This is a peer-reviewed, accepted author manuscript of the following research article: Terziev, Momchil ; Tezdogan, Tahsin ; Incecik, Atilla. / A posteriori error and uncertainty estimation in computational ship hydrodynamics. In: Ocean Engineering. 2020 .

\title{
A posteriori error and uncertainty estimation in computational ship hydrodynamics
}

\author{
Momchil Terziev $^{*}$, Tahsin Tezdogan ${ }^{1}$, Atilla Incecik ${ }^{2}$ \\ ${ }^{1}$ University of Strathclyde, Department of Naval Architecture, Ocean and Marine \\ Engineering, Glasgow, UK. \\ ${ }^{2}$ Faculty of Engineering, University of Strathclyde, Glasgow, UK \\ *Corresponding author: momchil.terziev@strath.ac.uk
}

\begin{abstract}
The increasing relevance of simulation-based design has created a need to accurately estimate and bind numerical errors. This is particularly relevant to full-scale computational ship hydrodynamics, where measurements are difficult and expensive, simultaneously requiring a high degree of predictive accuracy even in early design stages. However, the field of ship hydrodynamics has yet to fully exploit the enhanced capabilities and potential benefits numerical verification methods have to offer. The present study presents a detailed application of numerical verification procedures in CFD as applied to local parameters, such as free surface elevation and skin friction. This is done in order to pinpoint specific locations in the computational domain responsible for heightened levels of error and uncertainty. Relationships between different parameters are demonstrated and discussed based on a set of full-scale simulations of the KCS advancing through a canal using CFD.
\end{abstract}

Keywords: CFD, RANS, uncertainty, shallow water, KCS

\section{Introduction}

The year 2014 saw the publication of NASA's study on the future directions of Computational Fluid Dynamics (CFD) (Slotnick et al., 2014). Although their focus was predominantly on aerospace applications, several aspects overlap significantly with the field of computational ship hydrodynamics. This prompted Hawkes et al. (2018) to address one of the concerns raised by Slotnick et al. (2014), specifically, scalability problems of CFD simulations with increasing cell numbers.

A second key bottleneck, identified by Slotnick et al. (2014), is associated with solution uncertainty and robustness. This is directly related to the confidence levels one can attribute to a numerically derived solution. Such metrics are of critical importance in simulation-based design. Since computational power increases exponentially, it is reasonable to anticipate that Reynolds averaged, or other Navier-Stokes-based techniques will eventually become the norm in most forms of engineering analysis. Ship hydrodynamics is by no means an exception to this statement.

The rapid development in the prediction of flows around ships is exemplified in Figure 1, which was adapted from Witherden and Jameson (2017). The main difference between the present interpretation of the hierarchy of fluid flow models and that of the above reference is that Large Eddy Simulation (LES) has been incorporated at the top of the pyramid. This was done in light of recent publications on the matter, which demonstrated that LES can be used in practice, albeit subject to restrictions. An in-depth discussion on these can be accessed in the open 
literature (Fureby et al., 2016; Kornev et al., 2019; Kornev and Abbas, 2018; Liefvendahl and Fureby, 2017; Shevchuk and Kornev, 2017).

The issues, not addressed by Figure 1 relate to potential difficulties and their underlying sources relevant to each method. As one progresses towards the top of the hierarchy, different conceptual issues must be overcome. To elaborate, although the practicality of (for example) linear potential flow is not questioned, practitioners are aware of the situations where their use is admissible. The range of parameter definitions, computational resource and ease of implementation suggest that it is trivial to cross-reference solutions obtained with linear potential flow solvers to build confidence.

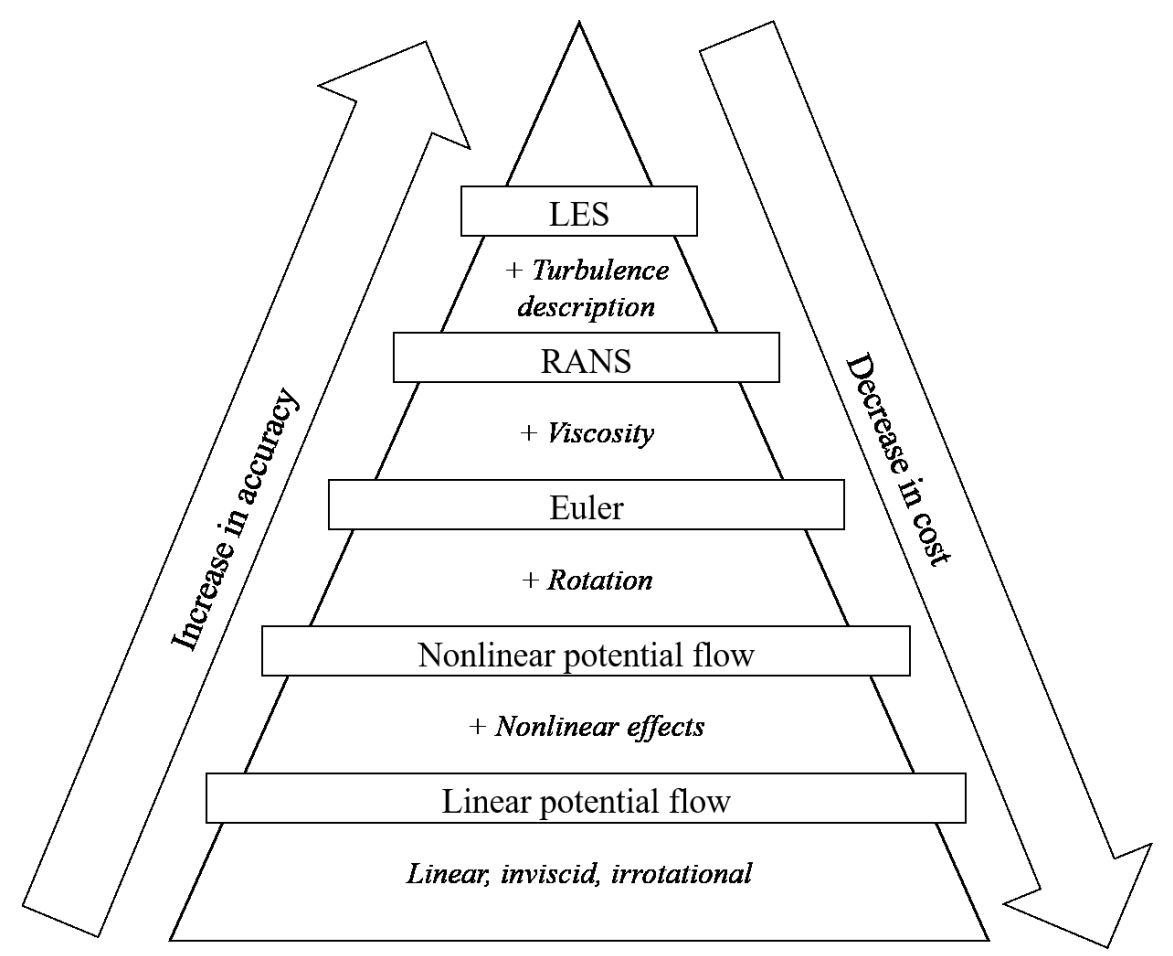

Figure 1. Hierarchy of fluid flow models, adapted from Witherden and Jameson (2017).

Near the opposite end of the complexity scale, RANS-based flow predictions feature dozens of parameters, models, sub-routines, and more generally, modelling approaches. These range from turbulence modelling, through boundary conditions, to numerical wave damping/definition, and interface tracking/capturing to name but a few. Each category mentioned above has emerged as a field of study in its own right, rendering it practically impossible for the practitioner to have adequate expertise simultaneously in all fields. For this reason, studies have emerged aimed at providing specific recommendations to alleviate the burden.

The required levels of technical knowledge can be reduced upon familiarisation with relevant research works for a wide spectrum of problems. However, one issue that remains challenging is that of grid generation. At present, constructing a mesh, onto which the solution of the governing Partial Differential Equations (PDEs) is to be obtained is as much an art as it is a science. This is true especially in cases where the requirements in terms of accuracy are high, as is often the case in ship hydrodynamics. Specifically, with ever tightening EEDI-related 
(Energy Efficiency Design Index) regulations, margins of error and uncertainty are slim. It is therefore imperative that once the practitioner has invested in performing what is currently the state-of-the-art in ship hydrodynamics, he/she can obtain an adequate margin of error associated with the solution. In other words, it is critical to determine the confidence one can place in the numerical solution, and discount specific results if necessary.

Lack of robust procedures to address confidence levels in numerical data that do not require user intervention (i.e. are automatically generated) is one of the key issues hindering in the large scale adoption of simulation-based design (Slotnick et al., 2014). This is particularly true of ship hydrodynamics which inherently features turbulent flows and fluid interfaces. These require knowledge of the simulation-specific phenomena, such as wakes and free surface deformations, where the computational mesh must be refined to capture the underlying physics well. Therefore, a key issue is related to mesh definition, which is almost always the greatest source of error (Eca and Hoekstra, 2006). Although grid sensitivity studies are frequently performed in an academic context, their outcomes are not always understood according to Salas (2006). In industrial applications on the other hand, the analyst may simply consider a single mesh due to the perception that error estimation is difficult and time consuming (Freitas, 2002).

This article will describe in detail, and demonstrate the use of a method developed by Cadafalch et al. (2002), and supplemented by Phillips and Roy (2017). This method utilises well-known procedures to predict the numerical error in the domain, simultaneously equipping the analyst with a measure of how well the grid has performed in reducing the numerical error. For the purposes of this study, full-scale simulations are sought in line with the fact that high Reynolds number flows are difficult to experimentally measure, and are where numerical work has the highest potential impact in simulation-based design (Stern et al., 2001). Specifically, the wellknown KCS was simulated in shallow water, replicating the experiments of Elsherbiny et al. (2019) in a rectangular canal at full-scale. Use is made of the commercially available RANS solver, STAR-CCM+, version 13.06.011 to perform the present analysis.

The remainder of this work will continue by introducing the necessary background in Section 2, followed by a description of the case-study in Section 3. The numerical set-up, and convergence properties of the solution are examined in Section 4, whereas the results and relevant discussion are presented in Section 5. Finally, concluding remarks, accompanied by suggestions for future research are given in Section 6.

\section{Background}

The accuracy of CFD methods is of great interest in academia for several reasons. When compared to analytical methods, computational approaches have a greater breadth of application and are capable of addressing significantly more complex physical phenomena (Oberkampf and Blottner, 1998). The main advantage of analytical methods is expressed in the fact that they are built on sound, reproducible and traceable mathematical arguments. However, this is simultaneously their major drawback. Many processes of immense practical importance, such as the turbulent motion of a fluid, cannot be described analytically (Durbin and Pettersson Reif, 2011). If this were possible, turbulence closures would be exact, and the field of turbulence modelling would not exist in its current form.

On the other hand, by avoiding the issues relating to analytical modelling of physical phenomena, the CFD method runs into a separate set of problems. These mainly relate to the 
equivalence between the continuum form of the governing PDEs and their discrete approximations. Lilek and Perić (1995) separate the errors into three categories:

1. Modelling errors, which can be thought of as the difference between the exact solution of the equations describing the fluid flow (i.e. satisfying the conservative laws), and the actual flow. For laminar flows, the Navier-Stokes equations are sufficiently accurate, but to account for cases where turbulence is important, additional models are required (Jasak, 1996). These errors are separate from the numerical errors discussed in this work (Oberkampf and Blottner, 1998).

2. Iterative errors, which arise as a result of the nonlinearity of the governing equations. The iterative fashion in which these are solved introduces errors, also known as iteration convergence errors (Eca et al., 2013).

3. Discretisation errors, which stem from the mapping of the continuum PDE and their related auxiliary models (for example, the turbulence model) into algebraic equations. Formally, this 'replacement' of equations rests upon Lax's Equivalence Theorem (LET) (Lax and Richtmyer, 1956). In essence, LET contains two statements regarding the discrete approximation of the PDEs. Firstly, the numerical procedure must be consistent, i.e. as the discretisation length (or mesh length $-h$ ) approaches 0 , the error must vanish. Secondly, the numerical method must be stable, i.e. if the one were to allow the solver to run indefinitely, the solution must remain bounded (Morton and Mayers, 2005).

Round-off errors are sometimes included in the above list. They can be traced to the finite digit storage on digital computers, but their influence is thought negligible (Roy, 2005). For this reason, we will not discuss their influence further. As mentioned above, modelling errors are not the focus of this work and are therefore discounted. Iterative errors are thought of as a simpler problem. In any case, their approximation is important because numerical error and uncertainty estimators have a tendency of magnifying solutions with large iterative errors, or incomplete iterative convergence (Larsson et al., 2014). For the adopted case-studies, these have been estimated in the region of $10^{-4} \%$ by using the procedure of Roy and Blottner (2006). Instead, the primary goal of this work is to apply to RANS-based ship hydrodynamics the discretisation error estimation procedures of local quantities and demonstrate their use. This is because the discretisation error category can account for more than $90 \%$ of all error (Xu et al., 2019). The use and application of discretisation error estimators is discussed in detail in the following sub-section.

\subsection{Discretisation error}

As stated previously, discretisation errors stem from the mapping of the continuum PDEs onto discrete locations. This is done by splitting the solution domain into a finite number of solution nodes. There are two methods to combat discretisation errors. The usual approach is to increase the density of space or time intervals, which the region of interest is subdivided into (although spatial discretisation is the focus of this work). This is typically referred to as spatial or temporal refinement, but cannot guarantee the solution will be improved. The above is exemplified in the findings of Larsson et al. (2014). In the aforementioned work on numerical hydrodynamics, solutions obtained with less than one million cells were compared solutions produced by $20+$ million grids from different participants. The graphical summary provided in Larsson et al. (2014) indicates that participants with low cell numbers often perform better than 
those with large cell counts. In other words, the arrangement, or properties of the grid are equally important in reducing errors (Salas, 2006). This is an issue that will be examined in the present work.

The alternative is to shift the focus from the subdivisions, explained above, towards equation discretisation. In the Finite Volume Method (FVM), which is used by Star-CCM+, the equation discretisation employed is $2^{\text {nd }}$ order. A second order discretisation approach is typical of RANS solvers, and implies that according to Lax's equivalence theorem, the error must reduce with the square of the grid size for asymptotic grids (Roy, 2005). In other words, the formal order of accuracy of the simulation is two $\left(p_{f}=2\right)$ (Roache and Knupp, 1993). Therefore, the second method to reduce the discretisation error would be to increase the formal order of accuracy, such as the $4^{\text {th }}$ order accurate method, devised by Lilek and Peric (1995). In practice, the choices in this respect are limited to $p_{f}=2$. That is, unless the user is re-coding the entire RANS solver. Consequently, the focus of this work will be on discretisation errors, stemming from grid density. The first method devised for this purpose is the Grid Convergence Index (GCI), devised by (Roache, 1998).

The GCI method predicts an uncertainty, which is used to bracket the numerically calculated solution. The true solution is then expected to lie within this bracket 95 out of 100 times, i.e. with 95\% confidence (Roache, 1997). The GCI method is based on Richardson's (1911) work, who devised a method to estimate a solution of fourth order accuracy based on second order finite differencing. Later, the approach, widely known as Richardson Extrapolation (RE), was improved upon by Richardson (1927). RE was originally formulated to use a refinement ratio $(r)$ of two $(r=2)$, which translates into grid doubling. However, it can be used with any factor larger than 1.1. That is, as long as the produced solutions are both sufficiently different, and asymptotic, to enable the validity of the method. This is in view of avoiding interference of other numerical errors (Roache, 1998).

The error of a numerical solution can be defined via a Taylor series expansion in the form shown in Eq. (1):

$\varepsilon=f_{\text {ex }}-f_{1}=\sum_{p=p_{f}}^{\infty} \alpha h^{p}=\alpha_{p_{f}} h^{p_{f}}+$ Higher order terms

where $f_{e x}$ is the exact solution, $f_{l}$ is the solution of obtained on a grid with characteristic size $h$, and $\alpha$ is a constant. In the present context, the error is defined as the difference between the exact solution and the solution obtained with a grid spacing $h$. In an attempt to reasonably approximate the error, higher order terms may be neglected. Thus, reducing the form of Eq. (1) into:

$\varepsilon \approx \alpha_{p_{f}} h^{p_{f}}$

The omission of higher order terms requires that their combined effect is much smaller than the error derived at the formal order of accuracy. The implications of this are that the grid must be asymptotic.

The reason why Richardson Extrapolation is not used per se is that it provides a $50 \%$ confidence level, whereas GCI boasts of $95 \%$, as stated earlier. This is achieved by magnifying the predicted numerical error by a Factor of Safety (FS). The value assigned to FS is clearly of 
high importance. In the GCI method, this is set as 3 when two grids are available, and 1.25 when three grids are used (Phillips, 2012).

Several methods have emerged in the recent past, many based on the GCI that attempt to modify the prediction of the FS. Some have made the FS a function of the observed order of accuracy, shown in Eq. (3) (Celik et al., 2008):

$p=\ln \left(\frac{f_{3}-f_{2}}{f_{2}-f_{1}}\right) / \ln (r)$

The fundamental notion is that if the observed and formal orders of accuracy are close, the grids are asymptotic. Otherwise, the GCI uncertainty diminishes rapidly with growing $p$, rendering unrealistically low numerical errors. Roache (1998) limited the observed order of accuracy to prevent this from occurring. Clearly, Eq. (3) can only be used when the argument of the natural logarithm in the numerator is positive and larger than 1. In other words, when the solutions exhibit monotonic convergence.

Unfortunately, the exact solution $\left(f_{e x t}\right)$ is known rarely, if ever, in fluid flows of practical interest. Using the order of convergence, one may re-write the error as a function of $p$ :

$\varepsilon(p)=\left(f_{2}-f_{1}\right) /\left(r^{p}-1\right)$

The uncertainty, $(U)$ is then simply the absolute error, as a function of the order of convergence, multiplied by the Factor of Safety:

$U_{G C I}=F S \times|\varepsilon(p)| /\left(r^{p}-1\right)$

Clearly, the Factor of Safety is characterised by a step change (1.25 to 3) within the framework of the GCI method. Stern et al. (2001) implemented a correction factor (CF) approach to resolve this. In essence, their approach consists of introducing a correction factor, used to account for higher order effects. This is used to magnify the error predicted via Eq. (4). In the above work, the correction factor is introduced as a metric describing the distance from the asymptotic range, where Eq. (4) is thought to be inadequate. Thus, the error according to Stern et al. (2001) takes the form of:

$\varepsilon_{C F}(p)=C \times\left(f_{2}-f_{1}\right) /\left(r^{p}-1\right)$

where $C$ is the correction factor, predicted as shown in Eq. (7):

$C=\left(r^{p}-1\right) /\left(r^{p_{f}}-1\right)$

Finally, the uncertainty can be estimated by:

$U_{C F}= \begin{cases}{\left[9.6(1-C)^{2}+1.1\right] \times \varepsilon_{C F}} & \text { when }|1-C|<0.125 \\ {[2|1-C|+1] \times \varepsilon_{C F}} & \text { when }|1-C| \geq 0.125\end{cases}$

Alternatively, the Factor for Safety approach of Xing and Stern (2010) uses the normalised order of convergence $(P)$, defined as the ratio of the observed and theoretical order of convergence $\left(P=p / p_{f}\right)$. Based on the value, attained by $P$, the uncertainty can be estimated as shown in Eq. (9):

$U_{R E F S}=\left\{\begin{array}{lr}(16.4 P-14.8) \times|\varepsilon(p)| & \text { when } P>1 \\ (2.45-0.85 P) \times|\varepsilon(p)| & \text { otherwise }\end{array}\right.$ 
All methods described thus far rely, in one form or another, on the observed order of accuracy. However, approximating an error does not require any knowledge of $p$. Indeed, $p$ is little more than the power, to which the grid $(h)$ is raised to in the dominant term of the Taylor series expansion, as shown in Eq. (1). Following such an approach, Roy (2008) split the problem into first and second order components. Thus, the fine solution $\left(f_{1}\right)$ can be expressed in terms of the extrapolated solution and a set of error terms as demonstrated in Eq. (10):

$f_{1}=f_{\text {exact }}+g_{1} h_{1}+g_{2} h_{1}^{2}+$ Higher order terms

In this approach, the constants $g_{1}$ and $g_{2}$ are defined as shown in Eq. (11) and Eq. (12), respectively:

$g_{1}=\left(r^{2} \varepsilon_{21}-r \varepsilon_{32}\right) /\left(r \times(r-1)^{2}\right)$

$g_{2}=\left(\varepsilon_{32}-r \varepsilon_{21}\right) /\left(r \times(r+1)(r-1)^{2}\right)$

where $\varepsilon_{21}=f_{2}-f_{1}$, and $\varepsilon_{32}=f_{3}-f_{2}$. Here, it is also useful to mention the convergence ratio $(R)$, defined as the ratio $\varepsilon_{32} / \varepsilon_{21}$, which is also the inverse of the argument in the numerator of Eq. (3). Armed with these parameters, the extrapolated solution may be estimated by:

$f_{\text {exact }}=f_{1}+\left(\varepsilon_{32}-\left(r^{2}+r-1\right) \times \varepsilon_{21}\right) /\left[(r+1)(r-1)^{2}\right]$

Roy (2008) defined the above procedure solely as an error estimator and did not provide a Factor of Safety, which can be used to predict an uncertainty. Therefore, one can make no claim with regards to the confidence level the method provides. To establish the range of applicability of the method, it must be applied to cases where exact solutions are known. This would allow the introduction of a Factor of Safety, which adequately accounts for the conservatism of the scheme.

The reason why this method is included in the present assessment is twofold. Firstly, the behaviour of the error can be examined with grid refinement, separating linear and quadratic terms. This permits one to determine whether the asymptotic range is approached from above, below, or from different directions by each term in the expansion (Eq. 10). The implications of such an analysis are important, because one may gauge whether the solution exhibits linear or quadratic convergence depending on which constant $\left(g_{1}, g_{2}\right)$ dominates in each range (coarse, medium, fine grids). The extracted information can then be used to assess whether further mesh refinement is warranted. This decision would rest on the approximated rate at which the error reduces. In other words, one may attempt to justify using a specific grid based on its convergence properties with greater ease than is possible with other methods.

For example, if a particular parameter exhibits second order convergence, the CFD practitioner may choose to refine the grid further. This could be a good choice since even a small reduction in the grid size can be expected to lead to a palpable reduction in the predicted error. Such tools are especially powerful when considering cases where experimental data are not available.

In second place, the spatial error, defined in Eq. (13), has third order accuracy, which is independent of the observed order of accuracy (Roy, 2008). Therefore, the method does not rely on the positivity of $1 / R$ in Eq. (3) and will produce an error estimate regardless of the parameters fed into Eq. (10-13). In practice, provided one has a greater number of solutions, a system of equations can be constructed in the form of Eq. (10). The first $n$ terms would allow the calculation of the $n-1^{\text {st }}$ constant $\left(g_{n-1}\right)$. Thus, when using three solutions, as is the case in 
this study, the linear and quadratic terms $\left(1^{\text {st }}\right.$ and $2^{\text {nd }}$ order $)$ are computed. Since the theoretical order of accuracy of the solver is $p_{f}=2$, there is little merit in seeking solutions of order 3 or higher.

It should be noted that a disagreement between the observed and theoretical order of accuracy does not necessarily imply inconsistency (Thomas and Langley, 2008). This is the case because Richardson Extrapolation was devised for structured grids. In fact, Diskin and Thomas (2010) point out that in unstructured grids, an order of accuracy near unity, or higher than two is frequently observed. They further state that this does not contradict the Lax and Richtmyer (1956) equivalency theorem, because the LET requirements are sufficient, but not strictly necessary to demonstrate the validity of a numerical scheme.

\subsubsection{Local error and uncertainty}

Having described the error and uncertainty estimation techniques, it is prudent to put into context the contribution of the present study. Here, the local error and uncertainty estimator, originally devised by Cadafalch et al. (2002) is introduced. The authors begin by defining Richardson and oscillatory nodes as follows:

- Richardson nodes: $\left(f_{3}-f_{2}\right) \times\left(f_{2}-f_{1}\right)>0$

- Oscillatory nodes: $\left(f_{3}-f_{2}\right) \times\left(f_{2}-f_{1}\right)<0$

Additionally, converged nodes can be computed, where the above product lies below some predefined measure of accuracy. For the purposes of this work, no such limit is defined. Therefore, it is thought prudent to proceed along the lines of Phillips and Roy (2012) and discount converged nodes as a possibility in the numerical work.

Cadafalch et al. (2002) then ensue to use the local observed order of accuracy to produce a global average. Following a similar rationale, Phillips and Roy (2017) provide an analogous framework, which is employed here. The first step is a small modification to Eq. (3), shown in Eq. (14). Specifically, the absolute value of the quotient in the numerator is taken. This ensures that oscillatory nodes are also taken into account. Such a change has been proposed previously in, for example, Celik and Karatekin (1997) and Celik et al. (2008). In other words, the change breaks no precedents.

$\hat{p}=\ln \left(\left|\frac{f_{3}-f_{2}}{f_{2}-f_{1}}\right|\right) / \ln (r)$

The resulting order of accuracy at each location of interest is not used directly. Instead, Phillips and Roy (2017) define the global deviation from the formal order of accuracy $\left(p_{f}=2\right)$, shown in Eq. (15):

$\Delta p=\min \left[\frac{1}{N} \sum_{i=1}^{N} \min \left(\left|p_{f}-\widehat{p}_{l}\right|, 4 p_{f}\right), 0.95 p_{f}\right]$

Eq. (15) can be interpreted as the mean local deviation of the observed order of accuracy from the theoretical order of accuracy. The maximum deviation is restricted to $4 p_{f}$ in an attempt to avoid skewing the average (Phillips and Roy, 2017). In the process of derivation of $\Delta p$, Phillips and Roy (2017) considered multiplicative factors of $p_{f}$ of 2, 4, 6, and 8, but the choice for this particular parameter was shown to be of little consequence. To prevent values close to zero, the maximum distance of the observed order of accuracy from the formal order is limited to 
$95 \%$ of $p_{f}$. Having obtained $\Delta p$, one can progress to calculating the global distance from the formal order of accuracy $\left(p^{*}\right)$, shown in Eq. (16). This enables the calculation of the Factor of Safety (FS), as a function of $p^{*}$, shown in Eq. (17).

$p^{*}=p_{f}-\Delta p$

$F S\left(p^{*}\right)=\left[F_{0}-\left(F_{0}-F_{1}\right)\left(\frac{p^{*}}{p_{f}}\right)^{8}\right]$

where $F_{0}=3$, and $F_{1}=1.1$. Eq. (17) can be used to construct a smoothly varying FS with distance from the asymptotic range.

Finally, the uncertainty can be estimated as shown in Eq. (18):

Uncertainty $=F S\left(p^{*}\right)\left|\frac{f_{2}-f_{1}}{r^{p^{*}}-1}\right|$

In the process of any RE-based method, a solution is extrapolated. This can be thought of as the solution, which one may expect to obtain on a grid with a cell size of 0 . In other words, it approximates the analytical solution. This is done by virtue of three systematically refined or coarsened grids. In this work, emphasis is placed on solutions achieved with uniform refinement ratios, although it is possible to vary this particular parameter. For the relevant background on this, the reader is referred to ITTC (2002) and Roache (1997). The use of an extrapolated solution as the final outcome of a simulation is inadmissible because it is not possible to prove that such a solution satisfies the conservative laws (Roy, 2008).

The methods presented thus far can be classified as a posteriori, because they allow the estimation of uncertainty after the simulation has run its course. However, there are a priori methods, which are, in theory, capable of performing the same function as soon as the grid has been generated. Since no such method is available in the RANS solver used, this course of action has not been explored further. Alternatives also include intrusive methods. For example, an error transport equation may be produced, which generates an estimate of the numerical accuracy while the simulation is running. In recognition of the fact that in practice, the average RANS practitioner will not be able to achieve this, the present study does not incorporate such methods. A full classification and discussion on the above methods can be found in Jasak (1996), Phillips (2014, 2012), Roache (1997) and Roy (2005).

In the remainder of this work, the case-studies and numerical set-up, and methodology are introduced. The necessary background is given on the selected case-studies.

\section{Case-studies and numerical set-up}

For the purposes of this work, a set of full-scale numerical simulations are used. These form a full-scale replica of the experimentally investigated case-studies of Elsherbiny et al. (2019). The refinement ratio $2^{1 / 3}$ was selected to avoid a sudden explosion in cell numbers. In other words, making this selection allowed the adopted simulation to have a greater number of cells while avoiding a sudden increase in cell numbers beyond which the simulations would become difficult to handle. The present simulations feature cell numbers ranging from approximately 10 million to 32 million. As explained previously, these cell numbers are achieved by a systematic, consecutive spatial refinement. 


\subsection{Spatial characteristics of the computational domain}

The dimensions of the computational domain satisfy two requirements. Firstly, the width and water depth are set to represent the experimental campaign of Elsherbiny et al. (2019), as shown in Figure 2. To reduce the required cell numbers, a symmetry condition is imposed, coincident with the ship and canal centreline. The width of the domain was originally $2.3 \mathrm{~m}$ in $\lambda=75$, whereas the depth $(h)$ was 2.2 times the ship draught $(h / T=2.2)$. Naturally, these conditions are maintained to reproduce the experiment as closely as possible in full-scale. To accomplish this, the side boundary is set as a slip wall, while the domain bottom is prescribed as a velocity inlet.

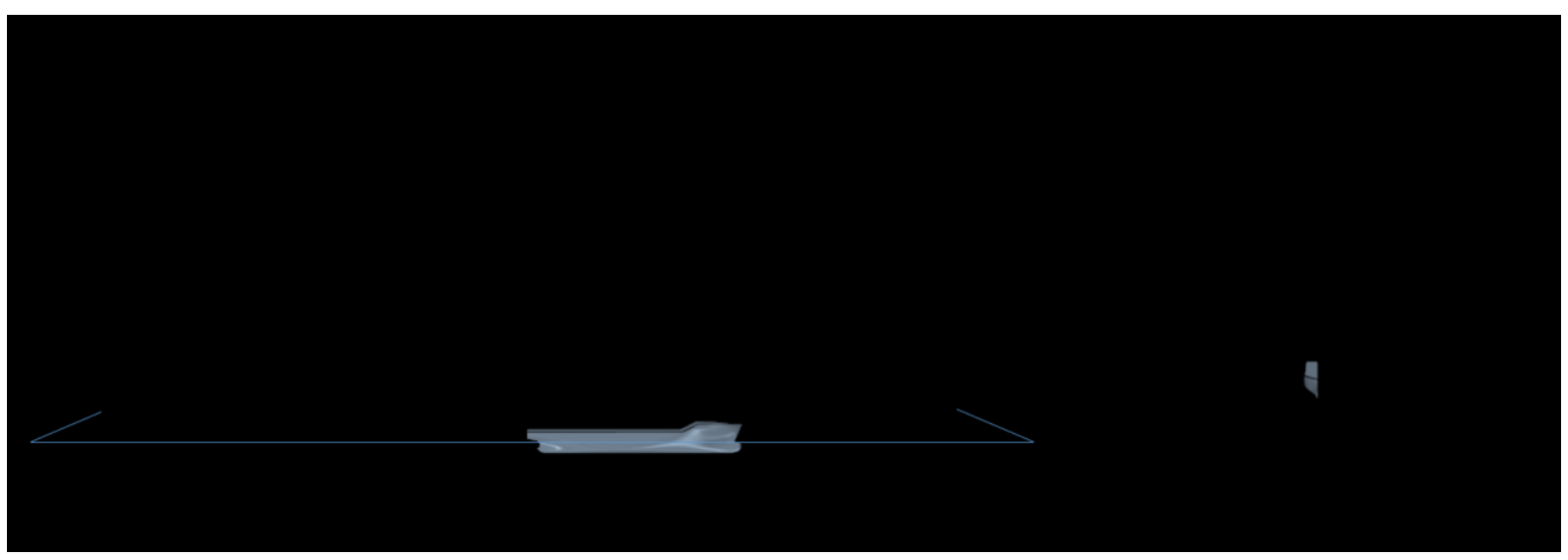

Figure 2. Domain dimensions and boundary conditions

The remaining boundaries are placed following widely used recommendations regarding resistance predictions in ship CFD. Specifically, the inlet boundary is positioned 1.5 ship lengths upstream of the forward perpendicular, whereas the outlet is placed 2.5 ship lengths downstream of the aft perpendicular (ITTC, 2014; Siemens, 2018). The domain top is set as an inlet, positioned 1.25 ship lengths from the undisturbed waterline. For reference, the ship and domain dimensions are shown in Table 1.

Table 1. KCS and computational domain principal characteristics in full-scale

\begin{tabular}{|l|l|c|c|}
\hline Quantity & Symbol & Value & Unit \\
\hline Scale Factor & $\lambda$ & 1 & - \\
\hline Length & $L$ & 230 & $\mathrm{~m}$ \\
\hline Beam & $B$ & 32.2 & $\mathrm{~m}$ \\
\hline Draught & $T$ & 10.8 & $\mathrm{~m}$ \\
\hline Depth & $D$ & 19 & $\mathrm{~m}$ \\
\hline Water depth & $h$ & 23.760 & $\mathrm{~m}$ \\
\hline Block coefficient & $C_{B}$ & 0.651 & - \\
\hline $\begin{array}{l}\text { Longitudinal Centre } \\
\text { of Gravity }\end{array}$ & $L C G$ & 111.593 & $\mathrm{~m}$ \\
\hline Wetted area & $S$ & 9530 & $\mathrm{~m}^{2}$ \\
\hline Speed & $U$ & 4.630 & $\mathrm{~m} / \mathrm{s}$ \\
\hline Reynolds number & $R e$ & $1.195 \times 10^{9}$ & - \\
\hline Depth Froude number & $F_{h}$ & 0.303 & - \\
\hline Water depth/Draught & $h / T$ & 2.2 & - \\
\hline Domain half width & $w$ & 172.5 & $\mathrm{~m}$ \\
\hline
\end{tabular}


All simulations were performed in the commercially available RANS solver, Star-CCM+, version 13.06.011. As mentioned previously, the software uses the Finite Volume Method (FVM) to discretise the governing PDEs onto a finite number of predominantly hexahedral cells with minimal cell skewness. The latter is defined as the angle between the face area vector (face normal) and the vector connecting the centroids of two adjacent cells. The reason why this parameter is important lies in the fact that diffusion terms contain the dot product of the two vectors referred to above (Siemens, 2018). Therefore, when these are perpendicular (i.e. the mesh is non-orthogonal), the diffusive terms are seemingly divided by zero, rendering severe convergence problems. The mesh must be as orthogonal as possible to avoid such a scenario.

In the free stream, there is little preventing the mesh from near perfect orthogonality. However, near the ship surface, the mesh must conform to the inherent curvature of the ship's underwater shape. This property, superimposed on the large aspect ratio cells, required to adequately capture boundary layer physics can create significant problems. The optimum aspect ratio of a grid was examined by Salas (2006), who concluded that it is possible to reduce the numerical error based solely on grid aspect ratio. In the present study, meshes with different aspect ratios are not manufactured, since this is too computationally expensive, considering the 32 million cells in the finest grid. Instead, the numerical error, as predicted for the finest solution will be examined against the aspect ratio of the cell, where the solution has been sampled from.

In the present study, two distinct metrics are assessed. The first is the free surface deformation, while the second - skin friction, acting on the hull. Since the ship was allowed to sink and trim via the Dynamic Fluid-Body Interaction module, offered by Star-CCM+, one cannot take free surface samples from the immediate vicinity of the ship hull. In the case of skin friction, the ship centre of gravity is used as the coordinate system origin, thus, automatically correcting for any differences resulting from the ship's squat. The sinkage and trim, computed from the three grids are shown in Table 2, while the resulting grids are shown in Figure 3. The free surface and skin friction are sampled at a simulation time of 500s to ensure all transient effects have decayed. Table 2 also contains the temporal uncertainty estimates, where arrived at by magnifying the time-step by a factor of $2^{0.5}$ to produce the medium $\left(f_{2}\right)$ and coarse $\left(f_{3}\right)$ solutions.

Table 2. Computed integral quantities and related uncertainties

\begin{tabular}{|l|r|r|r|r|}
\hline & \multicolumn{2}{|c|}{ Spatial uncertainty } & \multicolumn{2}{c|}{ Temporal uncertainty } \\
\hline Parameter & Sinkage $[\mathrm{m}]$ & Trim $\left[{ }^{\circ}\right]$ & Sinkage $[\mathrm{m}]$ & Trim $\left[{ }^{\circ}\right]$ \\
\hline$f_{1}$ & -0.186 & -0.0752 & -0.186 & -0.0752 \\
\hline$f_{2}$ & -0.186 & -0.0753 & -0.186 & -0.0753 \\
\hline$f_{3}$ & -0.187 & -0.0756 & -0.187 & -0.0754 \\
\hline$p[-]$ & 4.097 & 1.9643 & 2.450 & 0.7896 \\
\hline GCI $[\%]$ & 0.0220 & 0.1852 & 0.42 & 0.877 \\
\hline Convergence mode & Monotonic & Monotonic & Monotonic & Monotonic \\
\hline
\end{tabular}




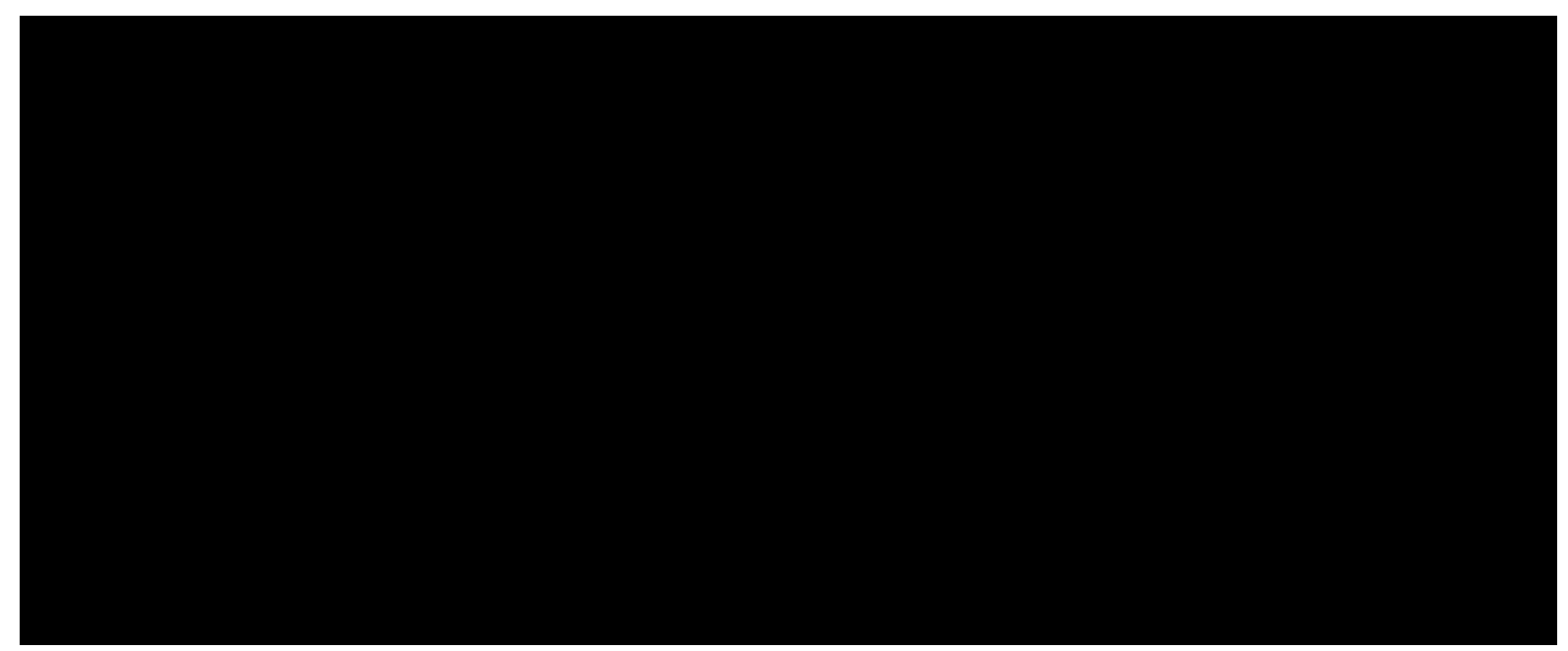

Figure 3. Resulting mesh

The mesh, depicted in Figure 3, was designed to capture the expected free surface disturbance by concentric volumetric refinements. This is done in the vicinity of the free surface, as well in the proximity of the ship hull. The top view of the mesh, shown in Figure 3 represents half of the computational grid. This is constructed within the automatic facilities Star-CCM+. The prism layer mesh is utilised to manufacture the near-wall mesh, which is accomplished by generating an exploded sub-surface, using the ship as input. Within the region between the subsurface and the ship hull, a fine mesh can be imposed in order to capture boundary layer physics.

\subsection{Physics modelling}

Typically, the near-wall performance of a simulation is characterised via the dimensionless distance from the wall, the $y^{+}$function. In model-scale computations, the desirable $y^{+}$values are smaller than $1\left(y^{+}<1\right)$. In full-scale cases, setting the $y^{+}$values below one is a significant challenge, involving very high aspect ratio cells. These are not a desirable feature in a numerical simulations, because they destabilise the convergence properties of the solution by introducing numerical stiffness (Deng et al., 2004; Eca et al., 2015; ITTC, 2011). It is also important to consider the transition of cell size. As the flow moves between two neighbouring cells, partial reflections may occur if there are large changes in the properties of the mesh (Siemens, 2018). Additionally, mesh coarsening introduces diffusion (Perić and AbdelMaksoud, 2016), which is not desirable in locations featuring near-wall influences. Fortunately, in full-scale applications, flow separation is less likely, and of less consequence. The question of whether employing wall functions amounts to correct flow features in the wake of a full-scale ship is yet to be determined. However, this requires experimental measurements in the wake of a full-scale ship, as well as the modelling of a spinning propeller, which is not within the scope of this study. The use of wall functions is therefore assumed justifiable (mean $y^{+} \approx 200$ in the present case).

The near-wall characteristics are governed by the distribution of velocity within the boundary layer. Naturally, this is dependent on the method, selected to close the Navier-Stokes equations. Such a model is required due to the fact that in their Reynolds averaged form, the governing equations do not form a closed set. For the purposes of this study, use is made of the standard $k-\omega$ model. This choice is made following recent work, conducted in the field by the present authors (Terziev et al., 2019), where the $k-\omega$ model was demonstrated to perform consistently 
and reliably. Moreover, the time per iteration was shown to be the smallest of all two-equation turbulence models assessed. Coupling these findings with the fact that the $k-\omega$ model can be applied seamlessly for any $y^{+}$value without any major modifications make it a good choice for the present assessment.

The importance of turbulence modelling in full-scale ship hydrodynamics has been discussed in Deng et al. (2004), who found that the non-linear Reynolds Stress Transport (RST) model exhibits best performance. However, the RST model suffers from several problems, not encountered in two-equation turbulence models. In particular, a greater number of equations is required to predict the Reynolds stress. Thus, the computational time is expected to be several times that achieved with the standard $k-\omega$ model. That is, albeit the solution of turbulence equations scales better than momentum equations (Hawkes et al., 2018). Although the RST model boasts of modelling turbulent physics more robustly than two-equation models, there are problems in its practical implementation (Parneix et al., 1998). The above suggest a conclusion along the lines of that made by Eca and Hoekstra (2001). Namely, two-equation turbulence models remain a good option in full-scale ship hydrodynamics. Moreover, the discrepancies between turbulence models are thought to be less significant with an increase in Reynolds number (Eca and Hoekstra, 2001), although more recent work suggests this may not be the case (Visonneau, 2005).

To guarantee that convective terms are represented as accurately as possible, a second order upwind scheme is adopted. This is done in view of the fact that upwind-biased schemes are the only available variety that can guarantee boundedness of the solution (Jasak, 1996). Moreover, adopting a higher ( $3^{\text {rd }}$ order) scheme can destabilise the solution because it introduces significant numerical diffusion (Vanka, 1987). The use of a second order accurate method is in line with the findings of Andrun et al. (2018). The aforementioned authors recommended the use of at least $2^{\text {nd }}$ order methods, although the results suggested little impact on free surface modelling. In the above reference, the authors also recommended the use of a least-squares (LSQ) approach to discretising the gradients. For all simulations performed here, the hybrid Gauss-LSQ method is used following the recommendations of software developer (Siemens, 2018). The definition of such a method is required because in addition to variable values, variable gradients must be computed at cell centres. These are used chiefly for the estimation of diffusion and convection properties.

The segregated flow method is used to solve the integral conservation laws in a sequential manner. This requires the solution of the three velocity components and pressure iteratively, one after the other. Velocity and pressure are coupled via a SIMPLE algorithm. Although the solver can cope with weakly compressible flows, the incompressible form of the governing equations is employed.

\subsection{Time step selection and temporal discretisation}

The temporal term in the Navier-Stokes equations is discretised via a first order method. This is done based the fact that the present class of problems falls within the 'pseudo-steady' category. To elaborate, the physical problem should not depend on transient terms per se. However, in practice, the RANS method requires the definition of temporal discretisation. Even though this may introduce an undue reliance of the computed results onto a metric they are theoretically independent of (Jasak, 1996). Time is advanced every 15 iterations by $0.0035 \mathrm{~L} / \mathrm{V}$ in the present simulations (note that this is smaller than the ITTC (2014) recommendation of 
$0.01 \sim 0.05 L / V)$. This choice for the time-step has been widely used and has been proven to provide sufficiently accurate results for similar cases (Terziev et al., 2019, 2018; Tezdogan et al., 2016, 2015; Zhang et al., 2018).

The reason why 15 inner iterations are used relates to residual reduction. Residuals indicate the degree to which the discretised equation is satisfied in each cell. Star-CCM+ uses the root mean-square value of the absolute error of all cells in the domain to provide a single metric (Siemens, 2018). Typically, one would seek residuals that are as low as possible, say in the range $10^{-6} \sim 10^{-8}$. In the present case, the specific dissipation rate was found to be satisfied to the greatest extent, achieving values in the range of $10^{-8}$. The remaining residuals, relating to the turbulent kinetic energy, $x, y, z$ momentum equations and continuity achieved values in the order of $10^{-4}$. The simulations were initially run with 10 inner iterations, but this was found to be insufficient to reduce the residuals to the desired range. This range is defined based on the recommendations of the ITTC (2014). Specifically, the residuals should decrease by $2-3$ orders of magnitude.

\section{Results and discussion}

The first step in this section is to present the input data required. As mentioned previously, for the purposes of this work, two different metrics are examined: free surface elevation and skin friction. The former is sampled $25 \times 10^{4}$ times, while the latter consists of $1.5 \times 10^{4}$ points on the ship hull.

The abovementioned points of the free surface are spaced uniformly in the $x-y$ plane on the starboard side of the ship and are depicted in Figure 4. Here, all spatial dimensions are normalised by ship length for consistency. The final sub-plot, shown in Figure 4 contains the convergence ratio $(R)$, whose extents are limited to the range of interest, namely \pm 1 . This is done due to the fact that if a point lands outside this range, the solution is divergent. From the figure it is immediately apparent that visual human perception of the differences with mesh refinement are by no means adequate to detect the discrepancies, highlighted by the convergence ratio. 

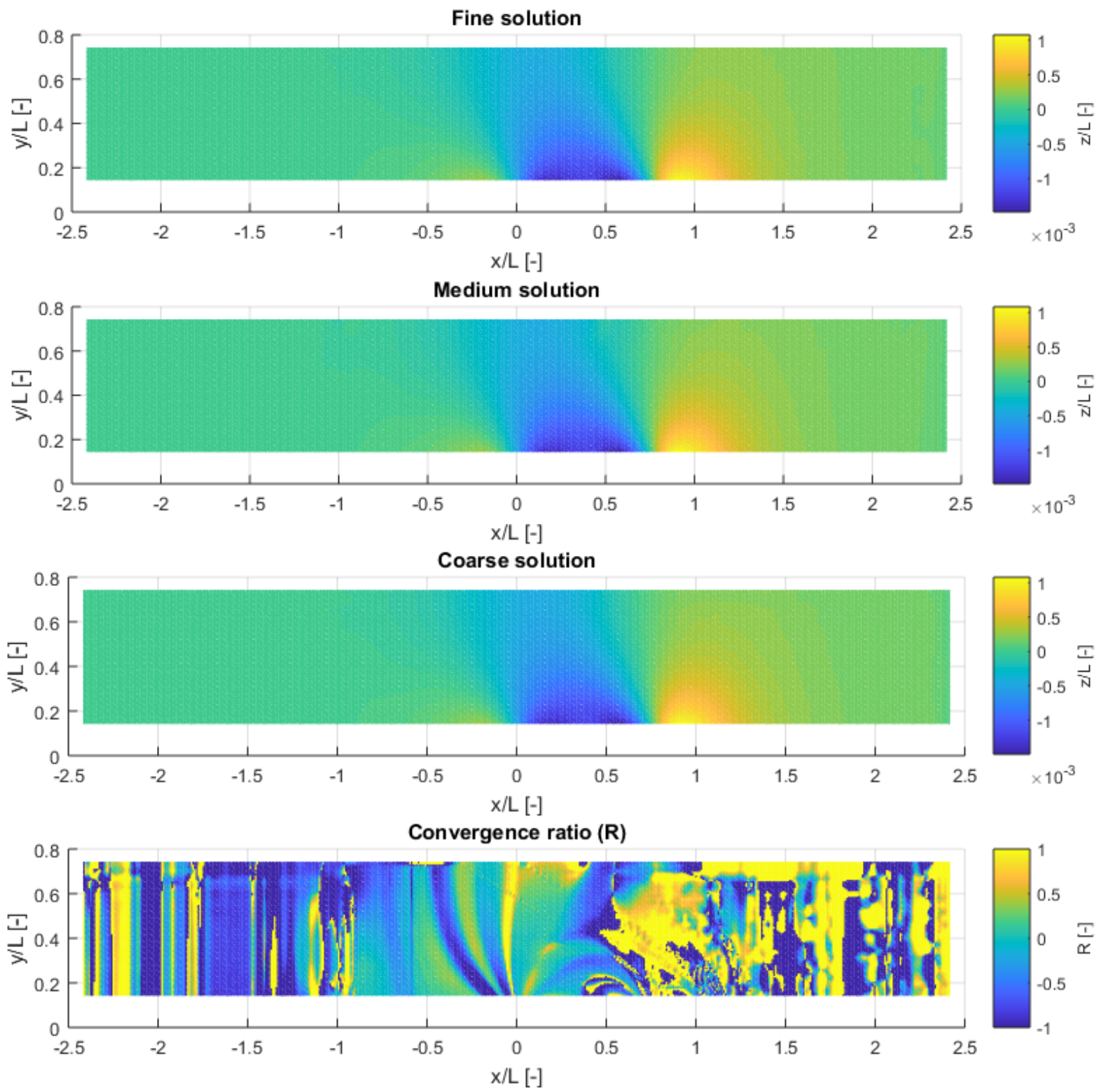

Figure 4. Fine, medium, coarse solutions, and convergence ratio of the free surface

It is important to mention that the use of the refinement ratio of $2^{1 / 3}$ means it is not possible to sample the free surface at cell centres of each grid, where the computations are performed. In cases where this is possible, the coarse grid determines the sampling locations, whereas the medium and fine grid are sampled every $r^{\text {th }}$ cell. In other words, the grids are nested. Instead, in the work presented herein, the RANS solver interpolates the free surface at the requested locations automatically. Such interpolation, and multiple sampling form a single cell in the coarse mesh may introduce additional errors. However, since the user is normally interested in an error estimator that is slightly leaning towards conservativeness, this is thought as an acceptable price to pay. In any case, an attempt to demonstrate the effect of sample density will be demonstrated later in this work.

In terms of skin friction, the ship hull is sampled at ten equally spaced lines, i.e. buttock planes. In the context of ship hydrodynamics, the underwater shape is typically the main contributor to resistance. Therefore, only the submerged underwater are is normally sampled for skin friction. However, if this were to be done, it would cause a different number of samples to be 
acquired from each grid. This is the case because even a small variation in the water surface near the hull may have a significant effect on the number of samples. For this reason, the entirety of the ship is taken. To eliminate the different sinkage and trim effect, the coordinate system is altered in each case to match the orientation and position of the centre of gravity of the ship. This allows all differences in position, orientation, and water elevation to be accounted for.

Moreover, a change in the free surface elevation in any single grid would cause a large numerical error and consequently uncertainty in the later stages. In other words, employing the here-described technique of sampling the ship hull for points, one avoids all potential problems, simultaneously accounting for disparities in the water surface elevation on the hull which are not addressed in the free surface sampling described previously.

The resulting points on the hull are shown in Figure 5, along with the convergence ratios. Points lying on the above water part of the ship naturally exhibit a much smaller skin friction than the submerged points, as expected. The former are found at the bottom of the plot when examining the distribution of skin friction in the longitudinal direction. It is also important to mention that all coordinates have been normalised by ship length, as was the case for the free surface.

In the present context, skin friction is defined via the the wall shear stress $\left(\tau_{w}\right)$. As before, the distribution of the convergence ratio in space reveals a complex pattern. It is clearly discernible that points near the parallel midbody may be responsible for significant deviations. This is an unexpected observation, since the curvature of the hull in this particular location is milder than it is near the more complex structures of the underwater shape, specifically, the bulb and the stern. 

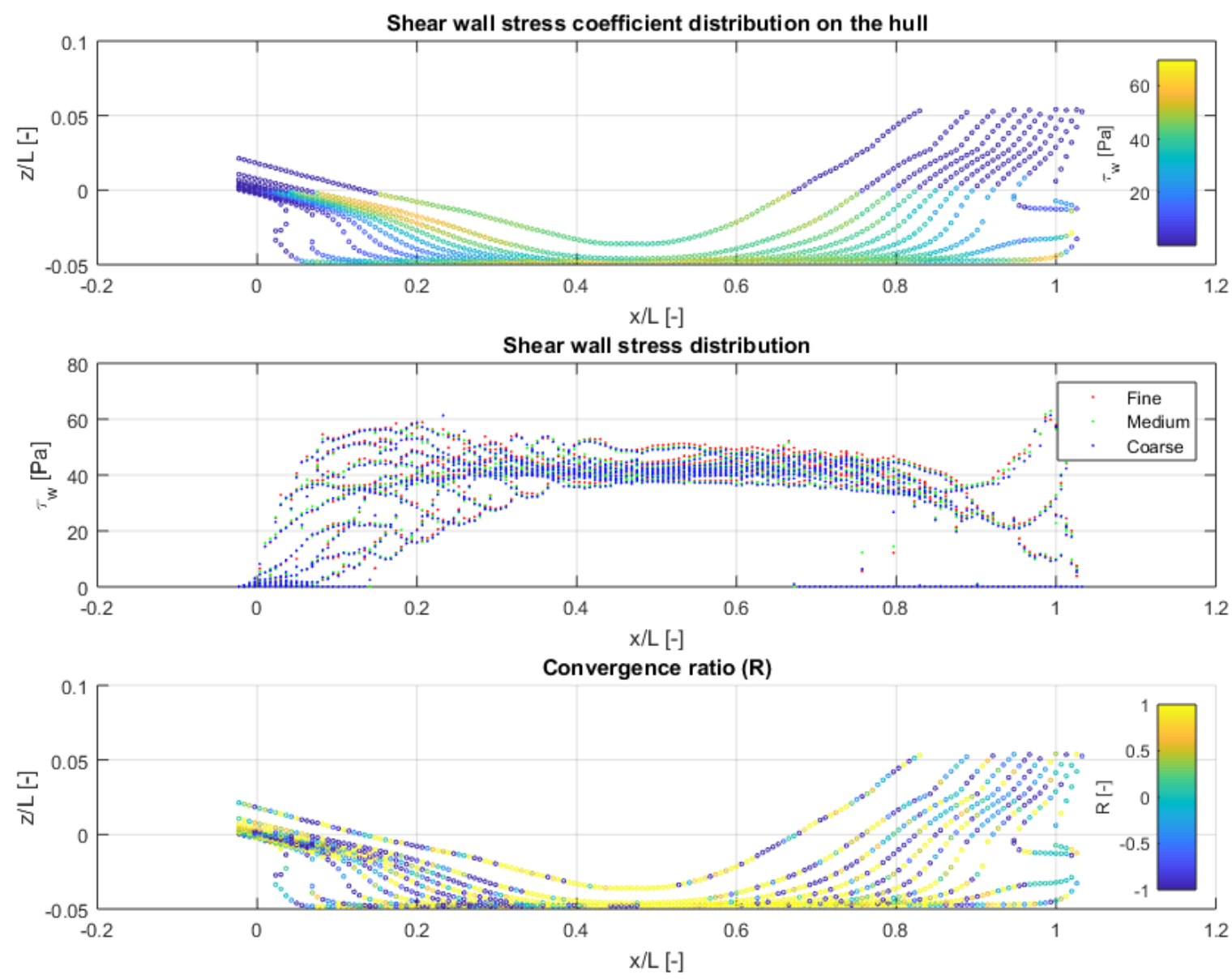

Figure 5. Skin friction nodes from the ship hull

\subsection{Local classification}

The next step is to determine which nodes can be classed as type 'Richardson nodes', and which are oscillatory. For illustrative purposes, this will be coupled with the local observed order of accuracy, as defined by Eq. (14). This is shown in Figure 6, where the Richardson nodes are predicted to account for approximately $43.8 \%$ of all nodes, i.e. circa $11 \times 10^{4}$ points in the case of the free surface. The remainder exhibit non-monotonic convergence or divergence. It is important to note that the local observed order of accuracy has been limited to 20. In any case, few points exceed this value. Figure 6 demonstrates that the computed free surface is separated into regions. The boundaries of these regions are characterised by a high observed order of accuracy.

The patches of Richardson or oscillatory nodes are clearly evident in locations, next to the ship hull, as well as up to half a ship length downstream. These locations are coincidently characterised by significant increases in mesh density (refer to Figure 3). The clearly distinguishable patches coincide with the wedge, prescribed to capture the potential effect of the Kelvin wake. In the present simulations, the low depth Froude number (0.303), coupled with the lateral restrictions allow for near-field waves only, as shown in Figure 4. 

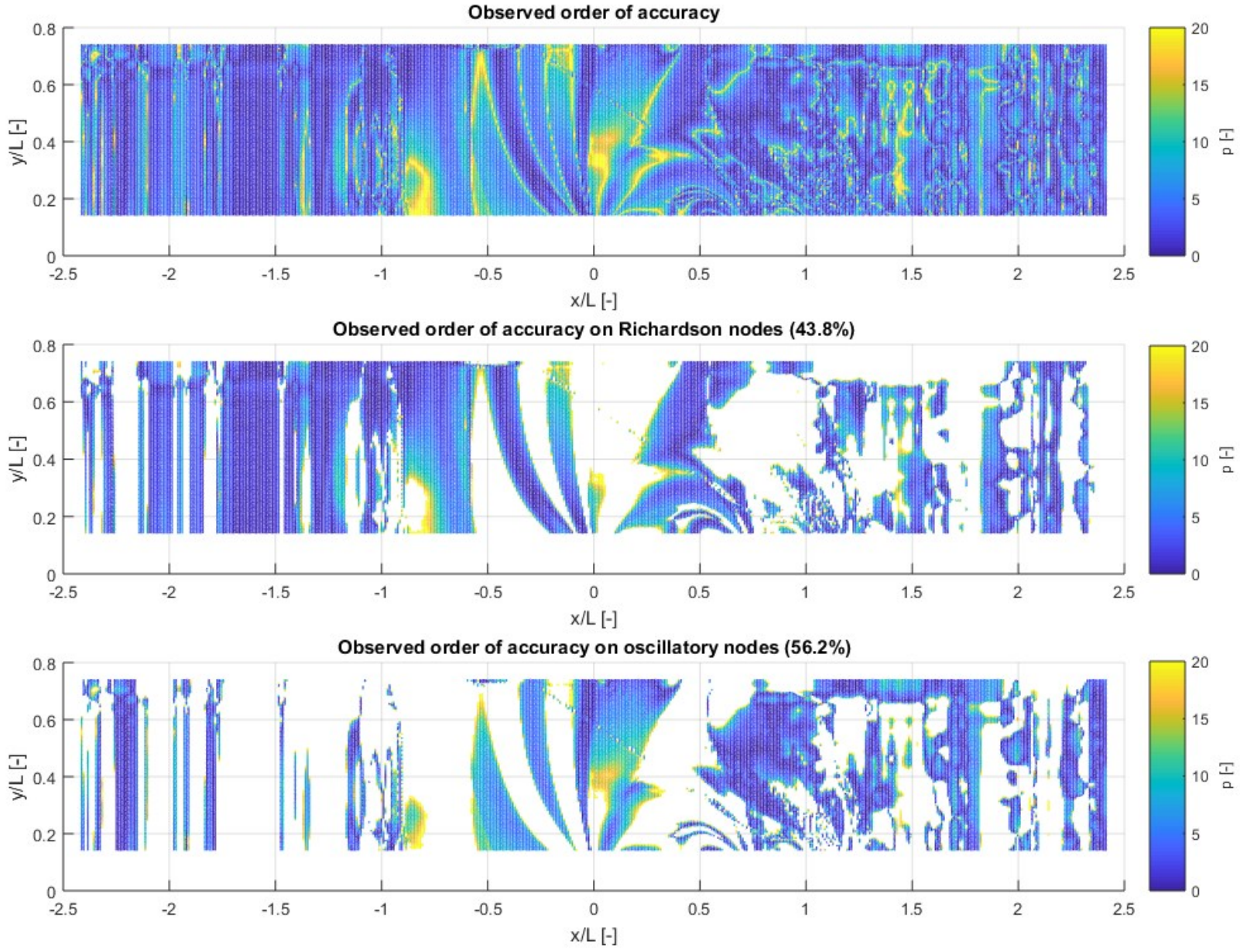

Figure 6. Observed local order of accuracy on the free surface nodes. 

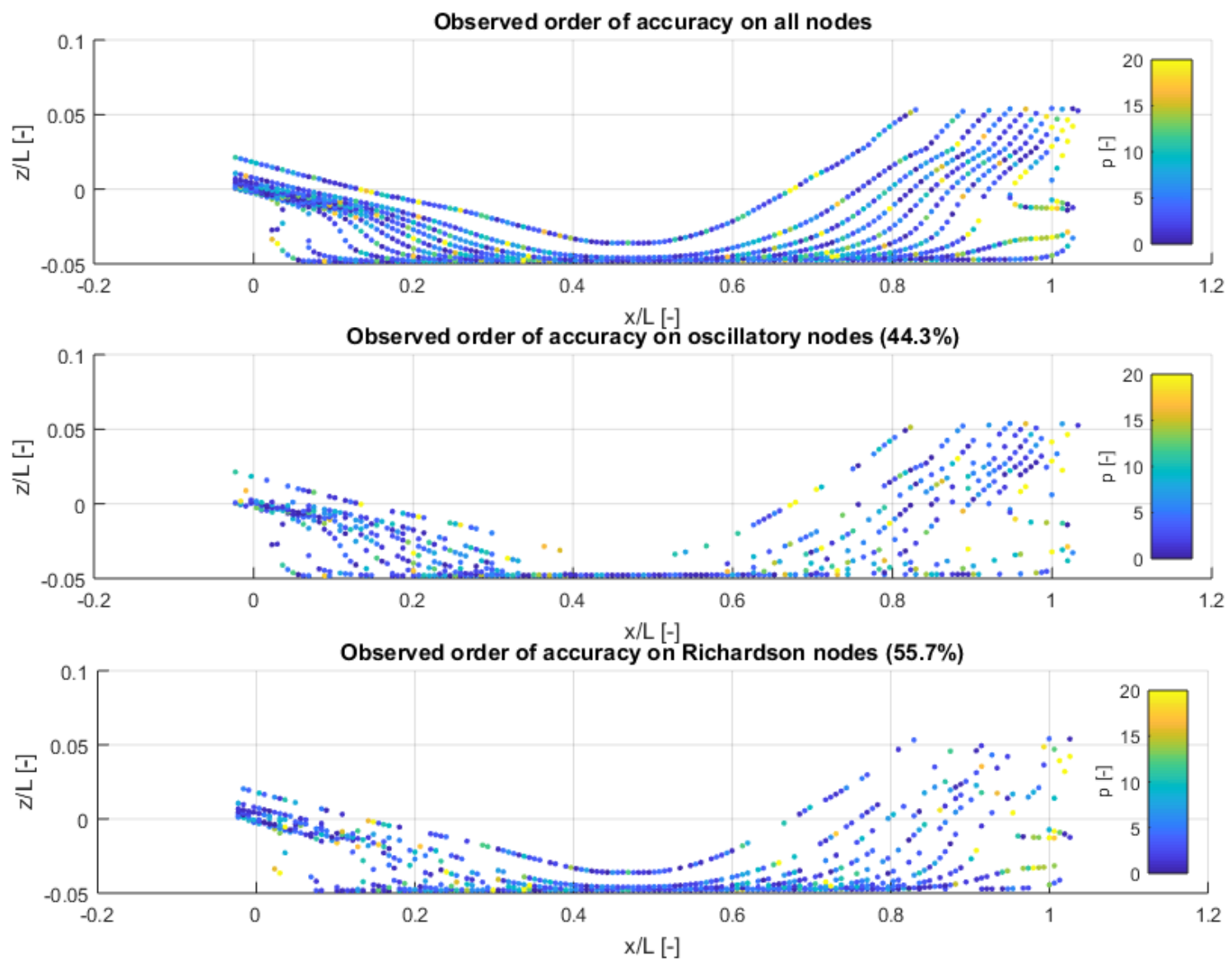

Figure 7. Local observed order of accuracy on the ship hull

The observed local order of accuracy based on the skin friction distribution on the ship hull is shown in Figure 7. Here, the maximum $p$ has been maintained as $p=20$ to retain consistency across the different parameters, examined in this study. However, as was previously observed, few points approach this limiting value.

The pattern of patches of similar behaviour seems to be largely maintained in Figure 7. To elaborate, it is apparent that nodes tend to switch from Richardson to oscillatory behaviour in patches along the ship hull, although this is not as easily observed as was the case in Figure 6. Many of the nodes previously highlighted as potentially problematic - near the parallel midbody of the hull - are shown to be characterised as of Richardson type. In the case of skin friction, fewer than half of all sampled nodes exhibit oscillatory behaviour. This shift, with respect the free surface highlights that different parameters may require separate consideration in examinations of the type presented herein.

\subsection{Error analysis and decomposition}

It is now prudent to examine the behaviour of the error constants on the free surface. These are calculated following the proposition of Roy (2008), and are shown in Eq. (11) and Eq. (12) for the linear and quadratic components, respectively. As stated in Section 2.1, there are two possibilities for the computed error constants. The $1^{\text {st }}$ and $2^{\text {nd }}$ order components either amplify or attenuate the overall error once summed. The first case can occur regardless of the sign of each constant, provided they approach the asymptotic range from the same side. The 
contribution of these nodes in the case of the free surface is demonstrated in Figure 8. Here, it is evident that the vast majority of error contributions are a consequence of linear and quadratic terms of opposite sign. Those with identical sign, i.e. approaching the asymptotic range from the top, or bottom are coloured in red, and account for a small fraction of the overall (less than $1 \%$ of all samples). Clearly, they can only exist in the narrow range where the two constants cross over to a different quadrant in Figure 8 . Thus, leaving the vast majority of nodes to be classed as having opposite linear and quadratic signs.

Therefore, all points, located near the extremities of Figure 8 contribute little to the overall error, and consequently uncertainty. In the same figure, the distribution of the error constants with respect to each other is also included. This is done to demonstrate the fact that most predictions are located near the centre of the plot. In other words, one should not expect excessive errors arising from misalignment of the linear and quadratic components of the error.

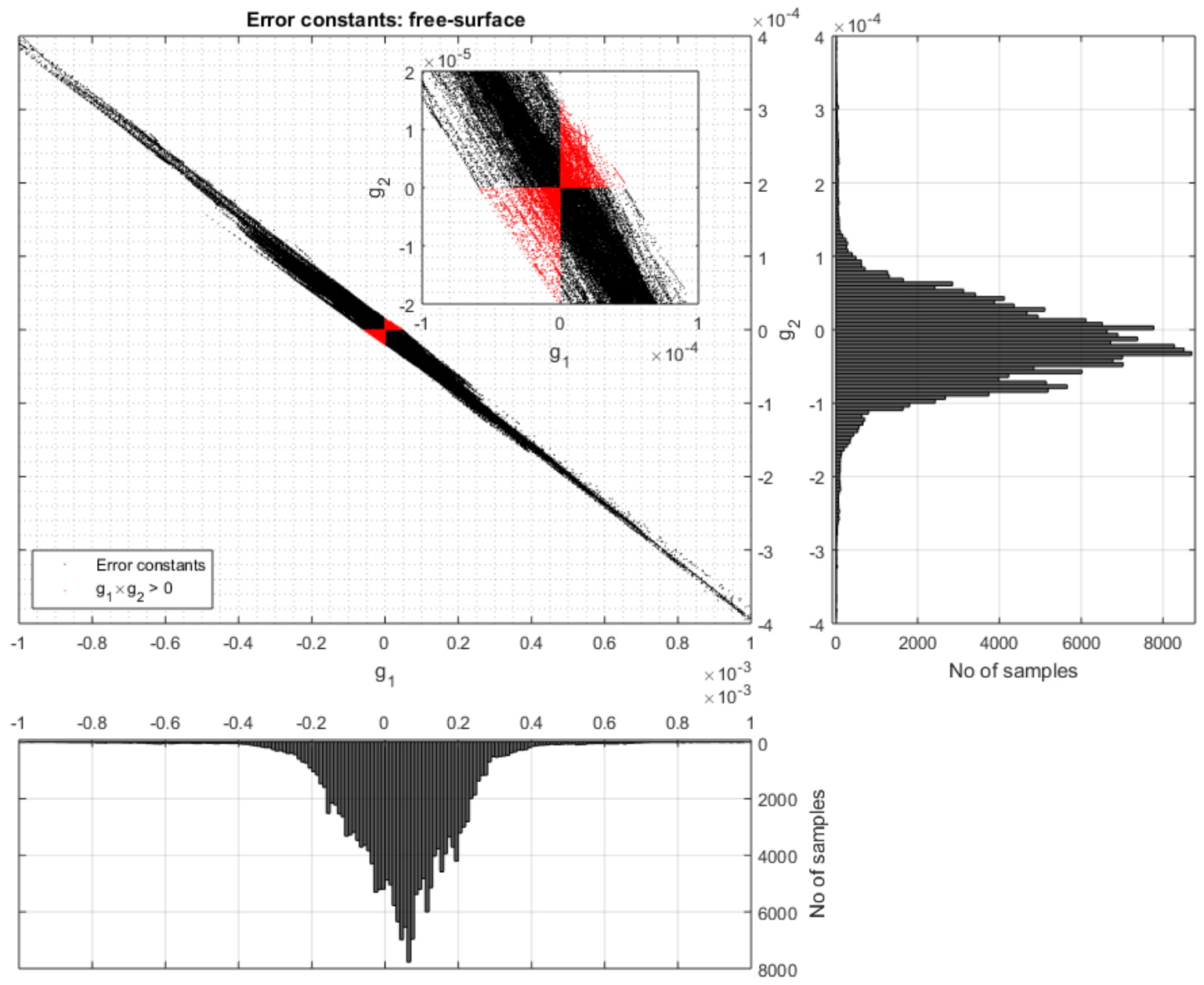

Figure 8. Free surface error constants.

In the case of skin friction distribution, the error constants magnitude is not similar to that of Figure 8, where the free surface error constants are given. The skin friction error constants are depicted in Figure 9, which replicates what was previously shown in the case of the free surface. Clearly, the number of samples in Figure 9 is significantly smaller than in Figure 8. Although this makes observations on the overall behaviour slightly more difficult, several general trends can be identified. 
In Figure 9, the range of both error constants is several orders of magnitude higher than was the case for the free surface points. This is a direct consequence of the fact that the latter were normalised by ship length prior being decomposed into $1^{\text {st }}$ and $2^{\text {nd }}$ order contributions. Therefore, the range of the axes should not be taken as an indicator of a problem in the assessment per se. Secondly, as was the case in Figure 8, the error is distributed along the diagonal of the plot in Figure 9. This observation, coupled with the small number of points where the error is reinforced in the process of summation has several implications.

Primarily, the alignment of points along the aforementioned diagonal of Figure 9 suggests that most points will exhibit a small error, resulting from the cancellation of linear and quadratic components. Additionally, the error amplifying points are constrained within a small wedge near the origin, implying a small numerical error can be expected to stem from these samples.
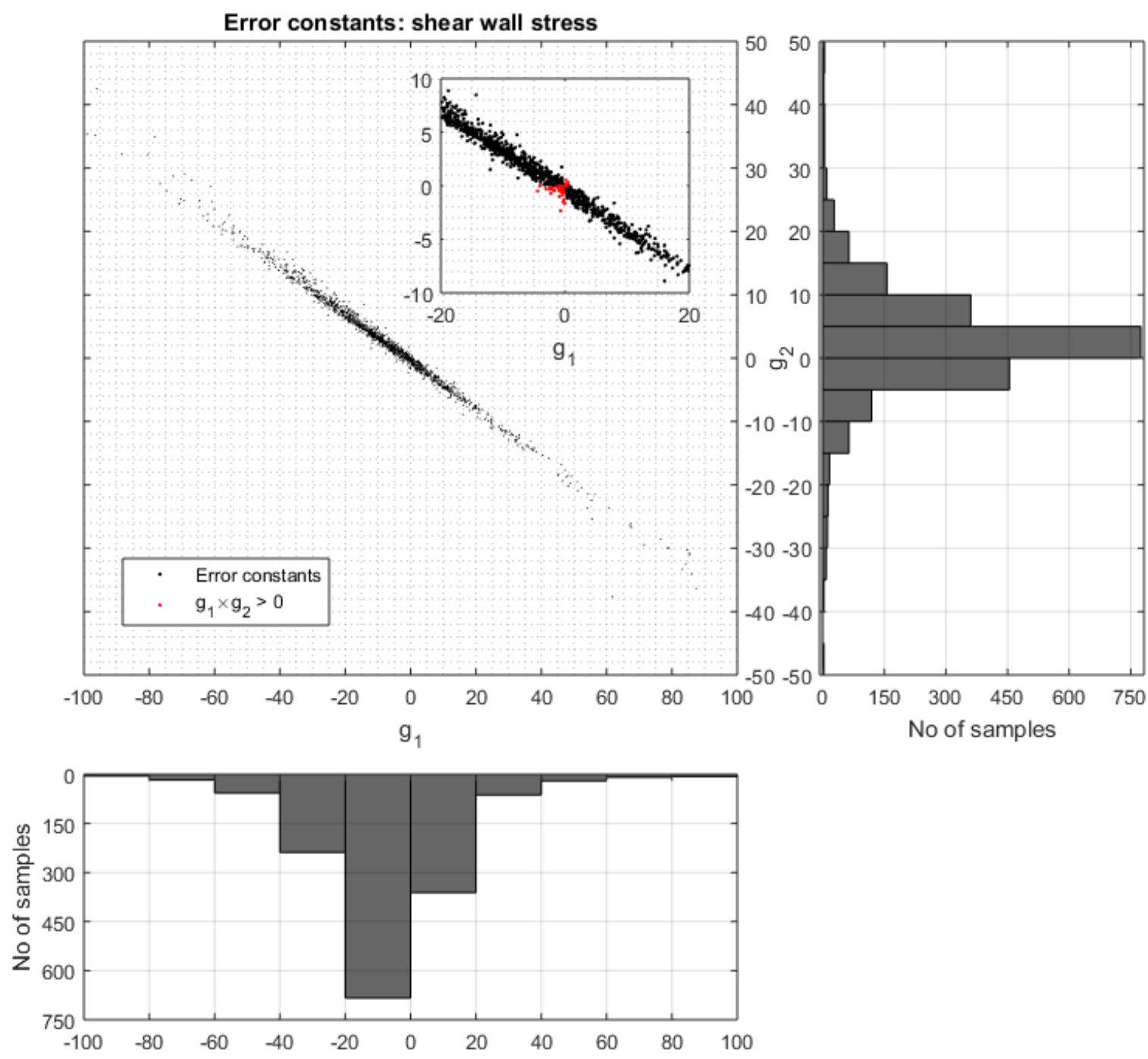

Figure 9. Skin friction error constants.

In the following section, the spatial distribution of the error is assessed for both parameters. For consistency, the free surface is examined first.

\subsubsection{Spatial distribution of the error}


The spatial distribution of the error constants (in the $x-y$ plane) is depicted in Figure 10 for the free surface. In the first sub-plot of the figure, the locations where the linear and quadratic components reinforce the overall error have been removed. With the exception of a line of samples near the outlet, the nodes where the error is amplified exhibit no discernible pattern. Thus, it may be postulated that such samples are a random occurrence, or are associated with a type of numerical error that is not known at present. In any event, the number of such points on the free surface is sufficiently small to be discounted.

The remaining sub-plots $\left(2^{\text {nd }}-4^{\text {th }}\right)$, shown in Figure 10 highlight the absolute values of the error constants. These allow one to pinpoint the specific locations, contributing to increased numerical error. Although any error estimation technique could have been used, Roy's (2008) breakdown is preferable because it allows a more detailed analysis. Moreover, since the same input is used for all uncertainty estimators, the predicted locations will remain constant, regardless of the method, as will be demonstrated later. The differences will arise in terms of magnitude, rather than relative error distribution in the domain. 

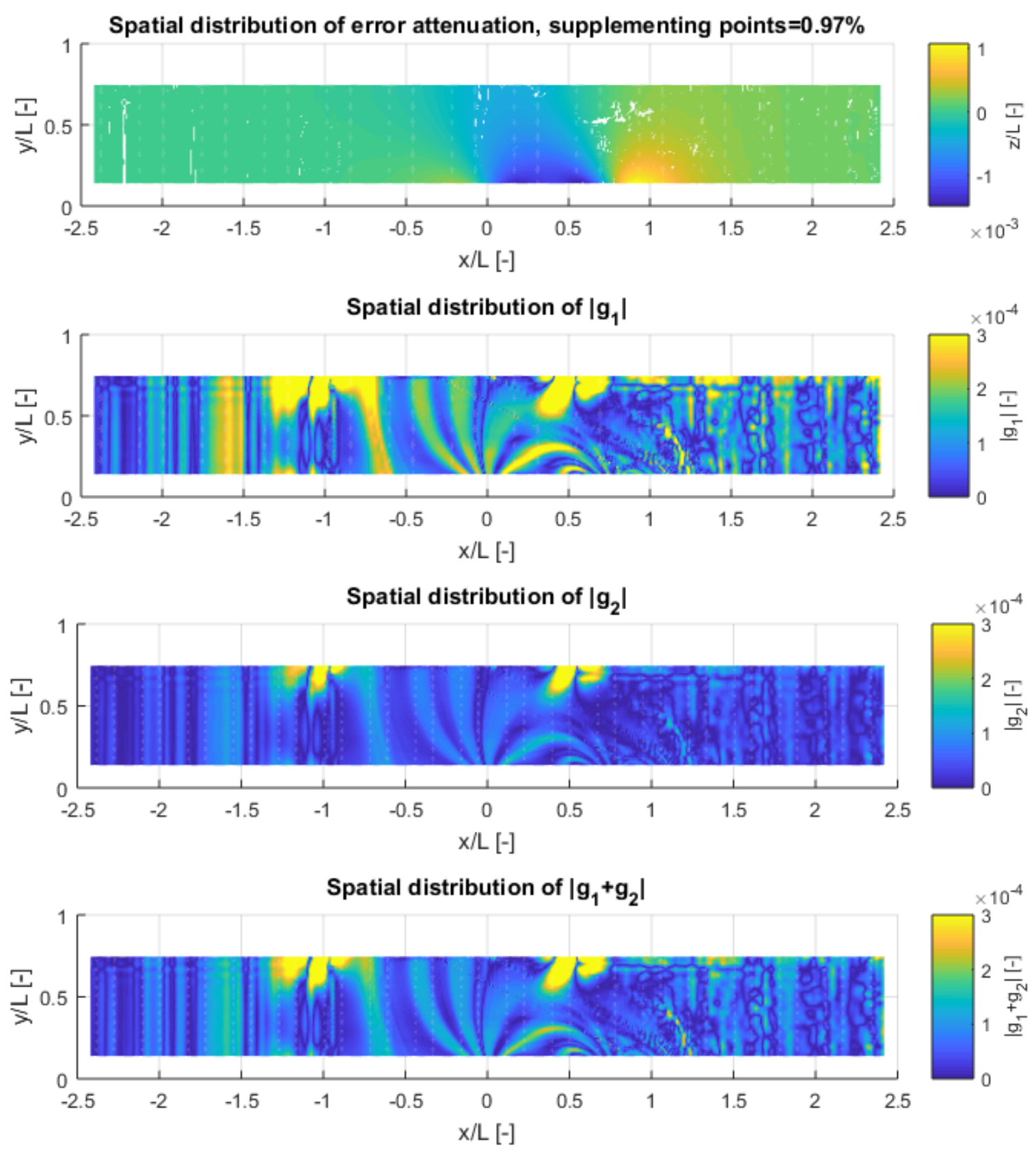

Figure 10. Spatial distribution of the linear and quadratic terms of the error in the free surface, computed via Eq. (11) and Eq. (12).

Figure 10 can also be examined from a different viewpoint. There are two distinctive features that are shown in all three possible error contributions $\left(1^{\text {st }}, 2^{\text {nd }}\right.$, and $3^{\text {rd }}$ order accurate solutions $)$. Specifically, the locations where the Kelvin wedge approaches the side wall exhibit elevated levels of numerical error. One may suggest that this is due to abrupt changes in mesh density. However, no such pattern can be seen over the remainder of the boundaries of the Kelvin wedge, whose arrangement can be consulted in Figure 3. Therefore, it may be speculated that the elevated levels of error are attributable to a combination of the change in mesh density, coupled with the proximity of a solid wall. In fact, such an observation is supported by the presence of nearly concentric semi-circles in the immediate vicinity of the ship hull (i.e. between $0<x / L<1$ ). 
However, the argument that an abrupt mesh density change in proximity of a no-slip wall may not be sufficient to describe the entirety of the 'error field' shown in Figure 10. In other words, the arcs of elevated error, located entirely within the Kelvin wedge do not fall within the above category. That is unless the interpretation of extended influence of the solid boundary onto the flow is adopted. To elaborate, it is possible that the hull's direct influence as a numerical error generator is greater than that of the side wall. This issue may require further study to accurately pinpoint the cause of these arcs.

The same argument in terms of method used to assess the error applies to the distribution of the skin friction on the ship hull, whose decomposed error distribution is depicted in Figure 11. As before, the first step is to examine the relative locations where the error is augmented versus those where it is attenuated. In the present case, 3.11\% of all samples fall within the former category - a significant increase when compared to the free surface. As was the case for the fee-surface, no discernible pattern can be identified in the spatial distribution of these points at present.

In Figure 11, the error constants are limited to a maximum magnitude of 60 to avoid skewing the axes too much because few points approach the limit. This also allows the identification of the points with elevated values with ease: almost the entire bulbous bow. On the other hand, locations near the parallel midbody show low levels of error. This is also the case for locations above the waterline. Such a remark may be interpreted as an indication that surface curvature creates elevated errors. To examine this claim further, the skewness angle of the first near-wall cell, along with its aspect ratio are shown in Figure 12.

Although significant skewness angles and aspect ratios are observed in Figure 12, no correlation can be identified between either of the above mesh properties and the observed error distribution. At the onset of the study, it was stated that an attempt is to be made to correlate the abovementioned characteristics of the numerical simulation. This was done in view of the fact that it has been demonstrated to be possible for simple cases (Salas, 2006). This may not have been achieved because a single mesh set-up was used and refined to produce the three solutions.

Therefore, as a piece of future work, the aspect ratio of near-wall cells, could be varied in a systematic manner to produce recommendations in this respect. A problem worth considering in the course of such a study would be whether the $y^{+}$values on the hull are targeted at the same value. In the event where they are, the change in aspect ratio must stem from a lengthwise reduction, i.e. grid refinement. Conversely, if the $y^{+}$values are not maintained constant across cases, the grid can be coarsened to achieve different case-studies. In the present case, the first near-wall cell's spanwise and lengthwise dimensions are a function of the base size. The base size represents the largest cell in the domain, and is found near the outlet and inlet. It is important to maintain the same aspect ratio across generated grids, because otherwise the procedure of Richardson extrapolation is invalid (Salas and Atkins, 2009).

Figure 10 and Figure 11 reveal two important aspects of the numerical simulation. Firstly, the linear contribution to the error is dominant in both figures. This observation, coupled with the fact that the majority of samples do not exhibit a behaviour of error amplification when summed suggests good performance of the numerical simulation. Such a conclusion can be drawn because the dominant error behaviour is known to be of $1^{\text {st }}$ order as the grid is refined 
past a certain point, and of second order in coarser grids. This is exemplified in the following sub-section.

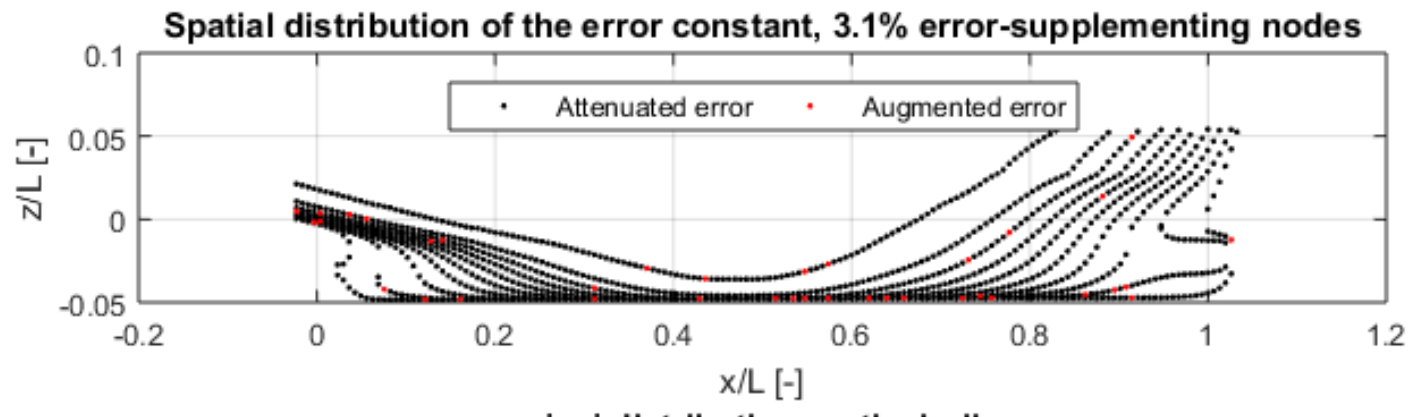

$\left|g_{1}\right|$ distribution on the hull

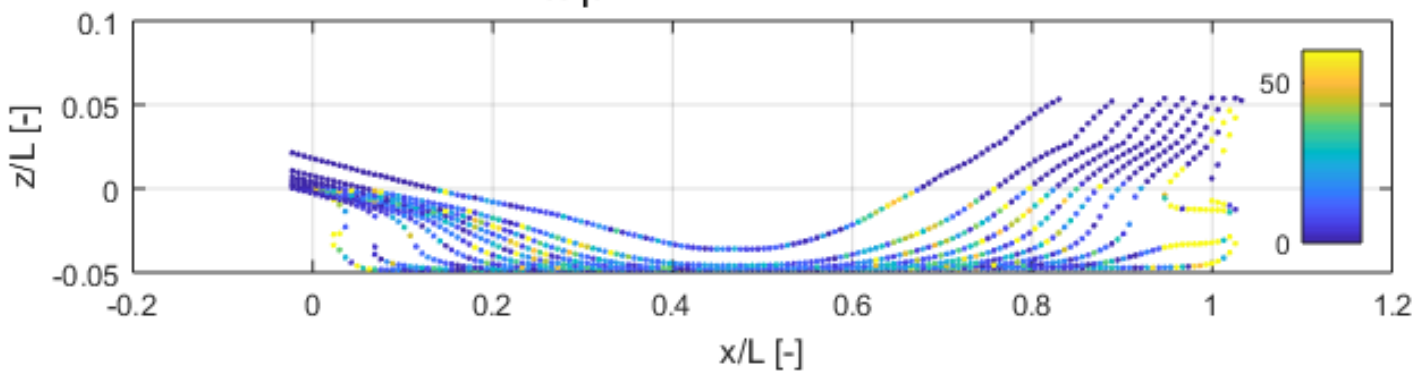

$\left|g_{2}\right|$ distribution on the hull

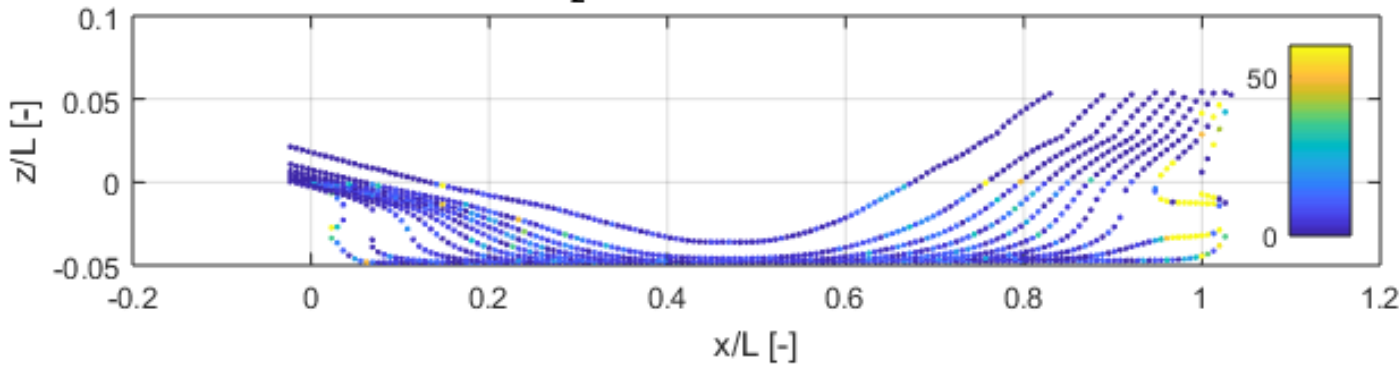

$\left|g_{1}+g_{2}\right|$ distribution on the hull

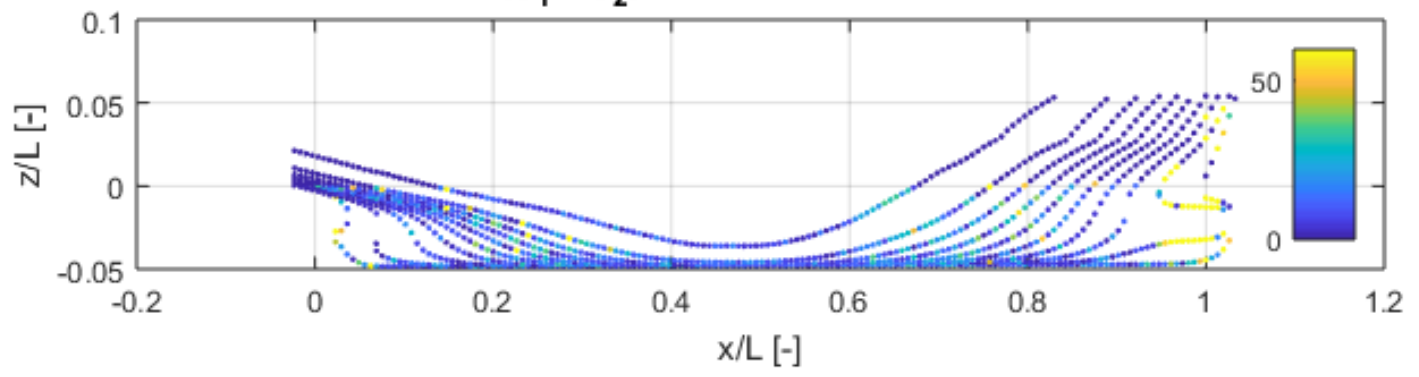

Figure 11. Spatial distribution of the linear and quadratic terms of the error in the skin friction, computed via Eq. (11) and Eq. (12). 

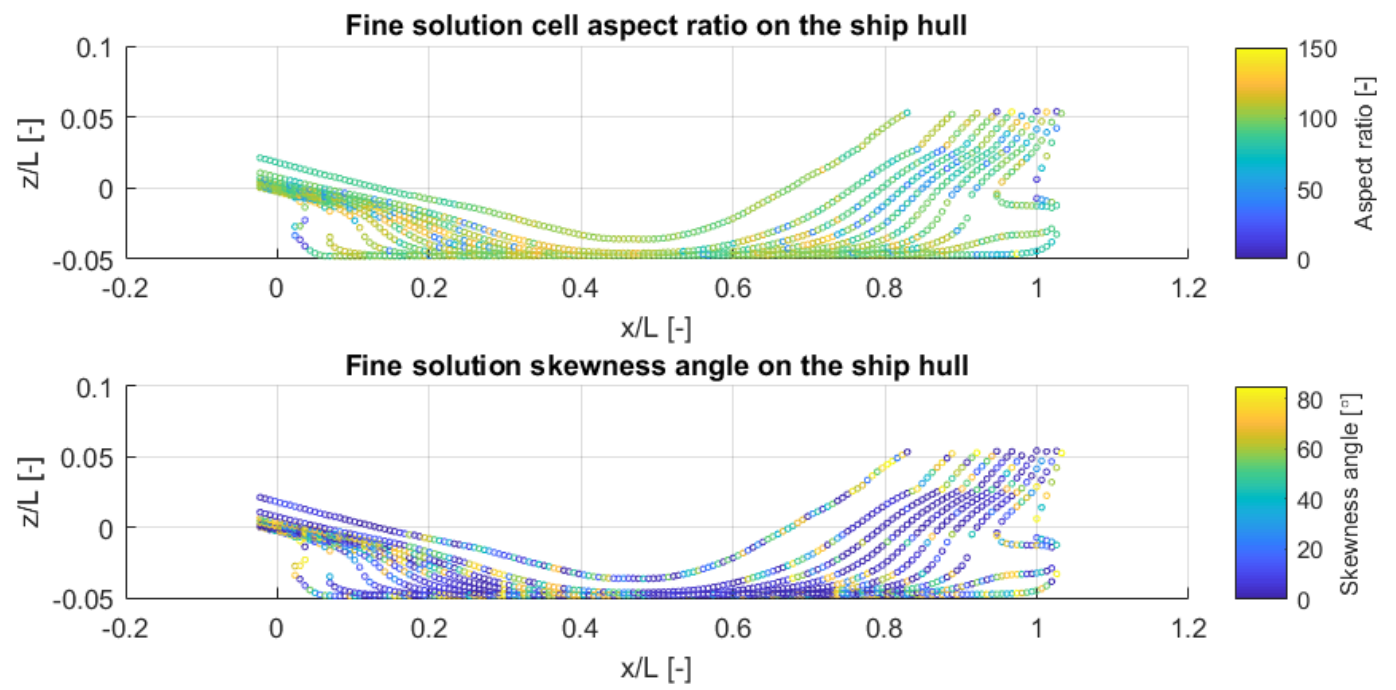

Figure 12. Cell aspect ratio and skewness angle on ship hull: fine solution

\subsection{Error behaviour considerations}

For this sub-section, samples from the free surface are considered only. This is done because of the greater number of available points. Regardless, the discussions and conclusions reached in what follows are equally valid for both parameters.

The error behaviour, shown in Figure 13 is assembled by randomly taking two samples from each error category depicted in Figure 8 . Specifically, a node where the $1^{\text {st }}$ and $2^{\text {nd }}$ order normalised error approach the asymptotic range from different sides, and a node where they approach the asymptotic range from the same side are extracted. These are classified with '-' and ' + ' in Figure 13, respectively. To simplify the figure, the logarithm of each term is used. This choice is made to allow the error line's slope to depict the order to which the term is raised. In other words, the line with slope one represents the first term of the Taylor series expansion shown in Eq. (10).

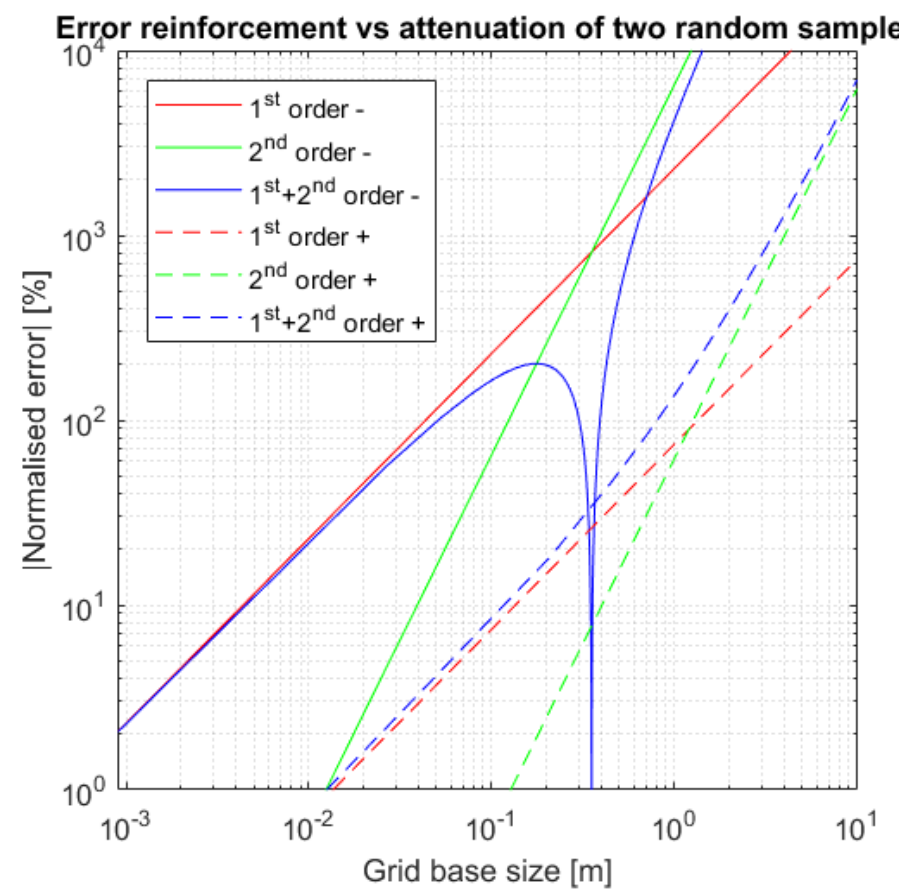

Figure 13. Example of error reinforcement and attenuation 
As referred to previously, Figure 13 depicts the presence of a hiatus when the first and second order error contributions intersect. This only occurs in cases where the asymptotic range is approached from opposite sides. Such a scenario is, as established in Figure 8, representative of the vast majority of all samples of the free surface and skin friction. Points near the hiatus are to be avoided, because of the possibility of spurious behaviour upon employing a grid in the proximity of the cusp. This is because Richardson extrapolation assumes smooth error derivatives, which is not the case in error attenuating scenarios near the cusp (Celik et al., 2005).

It is worthwhile to examine what would happen if, by chance, a solution were to be produced at precisely the grid size corresponding to the hiatus. In a typical Richardson extrapolation, for sufficiently fine grids the dominant error is raised to the lowest power, i.e. $p$. Thus, setting the order of accuracy as the lowest, dominant component of the Taylor series expansion and neglecting higher order terms is justifiable (Salas and Atkins, 2009). However, as $h$ is increased, multiple errors of different behaviour begin competing in the Taylor series expansion. Thus, setting $p$ as any one value and neglecting higher order terms is not correct in the vicinity of the hiatus.

The reason why Figure 13 is incorporated in this work is to demonstrate a fact concerning the error's behaviour with grid refinement. Specifically, second order convergence, or more precisely, error reduction, can only be expected for coarse grids. This is independent of whether the error is reinforced or attenuated by its separate contributions.

On the other hand, once the hiatus (if one is present) has been overcome, the cumulative error exhibits first order convergence. This follows as a consequence of the second order term's contribution decreasing at a much faster rate than the remainder of the error. Therefore, if one observes a global error with convergence properties that are close to linear, it can be claimed that the simulation is characterised by acceptable numerical error. It is important to note that the no claim of whether a particular grid is asymptotic or not is made based on the above criterion. Instead, it is suggested that little improvement, i.e. reduction in error can be achieved with grid refinement.

To elaborate, depending on the case, it may be justifiable to reject further grid refinement if the predicted numerical error will not decrease noticeably. Such an argument could be made if the error is shown to be located in the range where linear convergence dominates. On the other hand, if the specific grid produces errors in the quadratically convergent region, further grid refinement may be warranted. Further supporting evidence to this observation can be extracted from several works on numerical uncertainty estimation. In particular, the least-squares (LSQ) estimator of Eca and Hoekstra $(2009,2006)$. The LSQ method was designed to treat solutions with $0.5<p<2.1$ as asymptotic.

Another aspect of Figure 13 worth bearing in mind relates to the apparent order of convergence with grid refinement. It has already been stated that for fine grids, $p$ is unity, while for coarse grids, it attains a value of two. However, it is important to consider the manner in which such a transition occurs. In the case where the error is supplemented by the two components of the Taylor series, the error smoothly transitions from $1^{\text {st }}$ to $2^{\text {nd }}$ order. This can be easily deduced by an examination of the slope of the dashed blue line in Figure 13. There are no discontinuities, and its slope varies from 1 in the fine mesh region to 2 in the coarse mesh region. On the other hand, when the error is attenuated, the slope of the solid blue line exhibits a different behaviour. 
Although it too is of $1^{\text {st }}$ order in the fine mesh range, the transition to $2^{\text {nd }}$ order occurs though $-\infty$ and $+\infty$. In the course of this transition, the order of convergence attains all values except those between 1 and 2 (Salas and Atkins, 2009).

Combining the findings of Figure 10 and Figure 11 with those of Figure 13 suggests that the linear error has a much greater magnitude than its quadratic counterpart for the computed free surface and skin friction. Therefore, the locations where the majority of the error is expected to be found in the log-log plane of Figure 13 is to the left of the hiatus. That is, for most points, whose error approaches the asymptotic range from opposite directions. The error supplementing cases also follow a similar trend, albeit without the presence of a hiatus, which may produce spurious solutions.

In the context of asymptotic behaviour of the grid, there is one final angle from which the solution may be considered. As explained previously, the above method decomposes the error into first and second order components. Naturally, these are not dependent on the local observed order of accuracy, which is estimated via Eq. (14). This is true because $p$ (nor $\hat{p}$ ) is not used in Roy's (2008) method. However, the order of accuracy can be interpreted as a measure of the convergence mode. That is to say, if $p$ contains a real part only, then the solutions supplied into the equation converge monotonically. This is analogous to maintaining the convergence ratio between 0 and 1 .

To exemplify the behaviour of the error constants versus each component of a typical Richardson extrapolation, Figure 14 depicts the behaviour of the error constants against the convergence ratio, whereas Figure 15 - the error constants against the observed order of accuracy. Figure 14 is characterised by distinct asymptotes, located at a different $R$ value for each error constant. The prediction of where this location will lie depends exclusively on the refinement ratio. Specifically, no solutions can exist outside the boundaries defined by Eq. (20):

Asymptote locations $\left\{\begin{array}{lr}1 / r & \text { for } 1^{\text {st }} \text { order } \\ 1 / r^{2} & \text { for } 2^{\text {nd }} \text { order } \\ 1 /\left(r^{2}+r-1\right) & \text { for } 1^{\text {st }}+2^{\text {nd }} \text { order }\end{array}\right.$ 


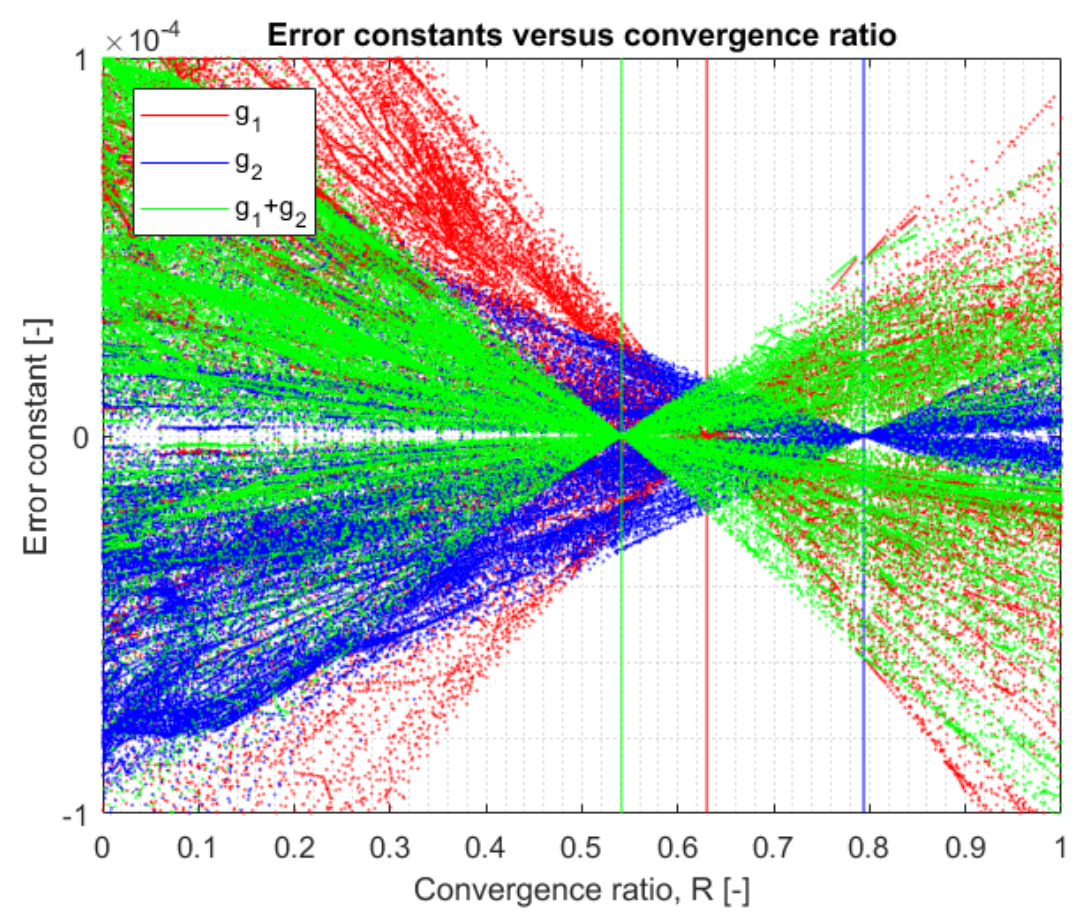

Figure 14. Error constants vs. convergence ratio

Having employed $r=2^{1 / 3}$, the asymptotes are located at $R=0.7937,0.6300$, and 0.5413 for the linear, quadratic terms, and their sum, respectively. Since one would ideally seek to obtain solutions that exhibit solely monotonic convergence, the $x$ - axis has been limited between 0 and 1 for $R$. Having established that the prediction of the relative location of the asymptotes is trivial, one could employ this information before conducting the numerical simulations. The choice of $r$ could be dictated so as to maximise the available area in Figure 14. In the present case, the choice of convergence ratio was governed exclusively by the rapid increase in cell numbers. Had a larger convergence ratio been selected, the cell numbers would have increased beyond a manageable size, whereas the idea was to allow for a large number of sequentially refined sets of simulations to be achieved.

Figure 15 demonstrates that although Roy's (2008) method does not explicitly depend on the order of accuracy, discernible patterns can be identified by combining the two. The error constant's behaviour on monotonically convergent samples shows clear trends, whereas the non-monotonic nodes do not. The former are characterised by a sharp cusp. The linear component shows that this cusp is located at $p=2$, the quadratic at $p=1$, while their sum: $p=2.654$. Figure 15 also confirms that the error due to the $2^{\text {nd }}$ order term attains a smaller magnitude over the entire range examined. The $1^{\text {st }}$ order term also grows at a greater rate than the quadratic term as the distance from the cusp is increased. The relative location of where this occurs is again governed exclusively by the refinement ratio. To demonstrate this, one may simply substitute the terms of Eq. (20) into Eq. (14). In the non-monotonic case, all points seem to have a tendency of increased error when compared to the monotonic samples. Therefore, despite the fact that the method does not take into account the convergence ratio, nor the order of accuracy per se, clear relationships can be established based on these components and the error constants. 

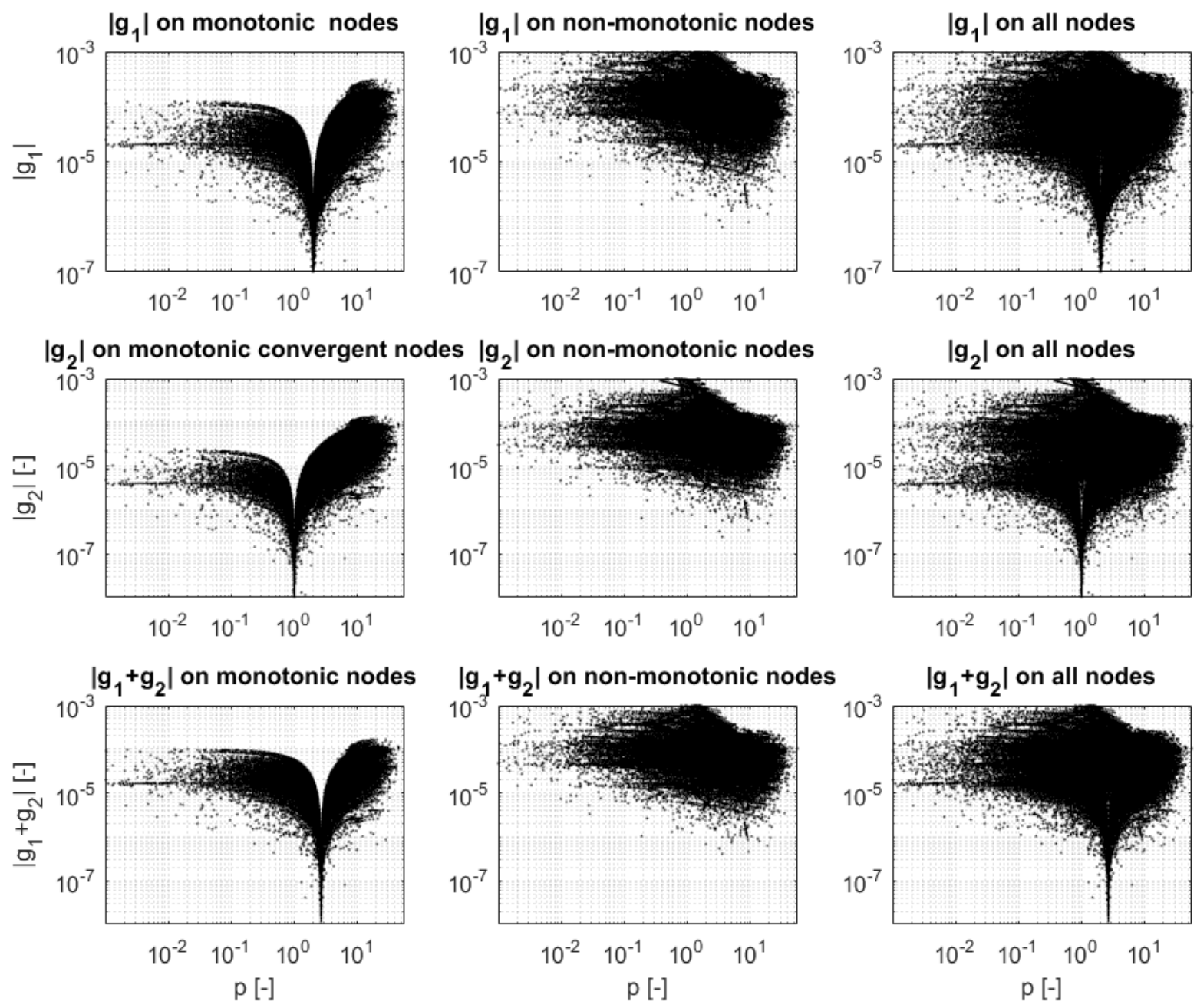

Figure 15. Error constants vs. local observed local of accuracy

\subsection{Local uncertainty and extrapolated solutions}

In this sub-section, the uncertainty estimates are presented for both the free surface and the skin friction data. A discussion is also given on the choice of the Factor of Safety, as well as the best choice for the order of convergence.

\subsubsection{Factor of Safety}

The first step is to determine whether the suggested FS of 1.25 as part of the GCI procedure agrees with the procedure of Phillips and Roy (2017) for estimating the FS. This is presented for both local parameters in Figure 16.

The results reveal that the skin friction data is asymptotic based on the criterion of Eq. (17), which exhibit a Factor of Safety of 1.2532 with a distance from the asymptotic range of 0.0209 . The reader is reminded that in the present context, the distance from the asymptotic range metric is defined as the deviation from the formal order of accuracy $\left(p_{f}=2\right)$. Therefore, the skin friction data is highly asymptotic, suggesting that further refinement is of little use in the present case. This is an encouraging finding considering the high aspect ratios of the near-wall cells and their skewness angles, shown in Figure 12. 
The free surface on the other hand is highly non-asymptotic. It may seem that the point depicting its relative location on the $\Delta \mathrm{p}$ range is not at its further possible limit. However, Eq. (17) limits the distance from the asymptotic range to $95 \%$ of $p_{f}$, i.e. a maximum of 1.9 , where the free surface Factor of Safety lies. If this limit were not imposed, the attained value along the $\Delta \mathrm{p}$ axis is predicted as 3.0571 .

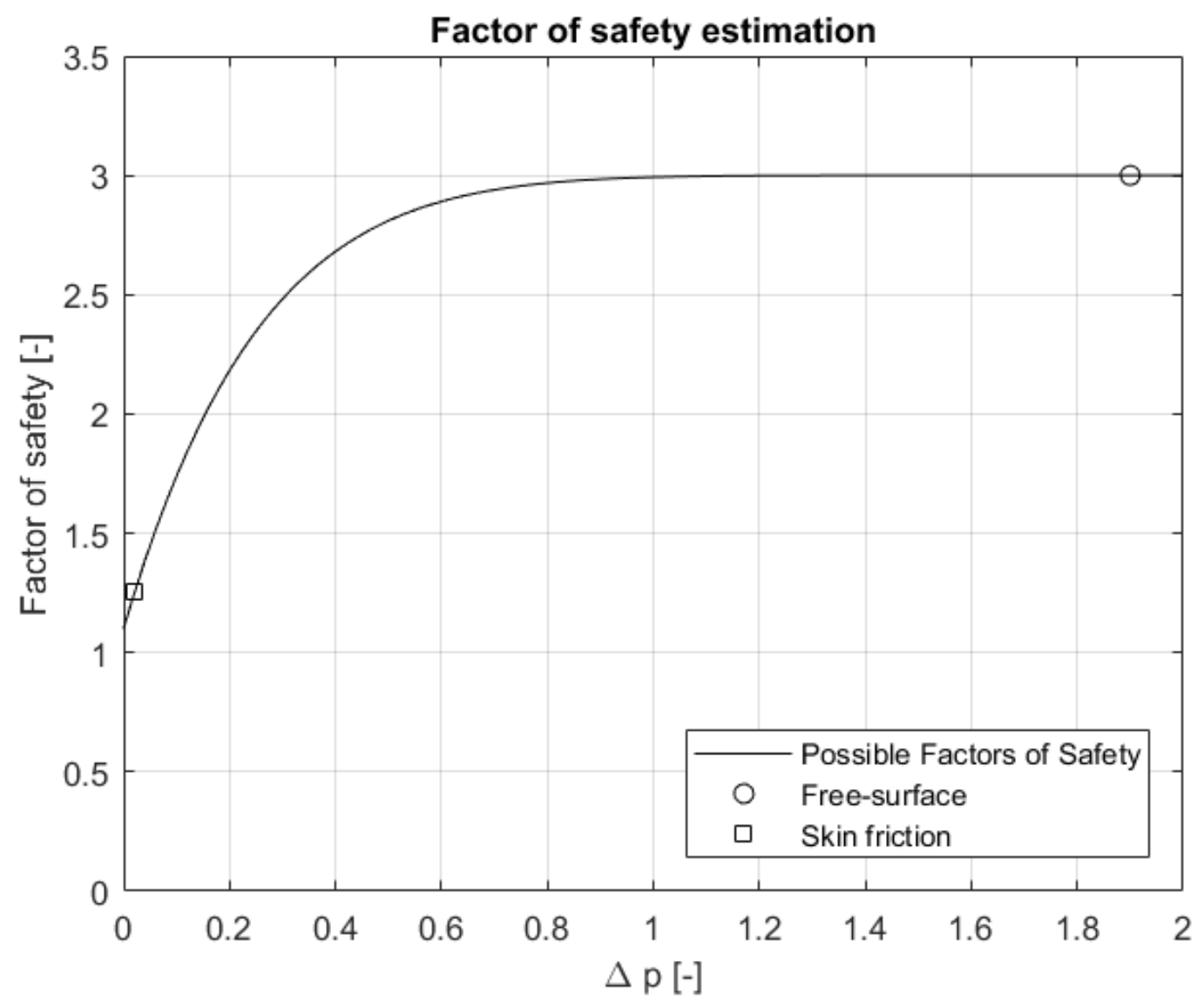

Figure 16. Computed Factors of Safety using Eq. (17).

The remainder of the factors of safety and modified orders of convergence are not shown because they depend on simple manipulations of $p$ and $p_{f}$. Instead, prevalence is given on the uncertainty itself in what follows.

\subsubsection{Uncertainty spatial distribution}

In this sub-section, the uncertainties for both sampled parameters are given. This is done according to all methods described in Section 2.1.

In Figure 17, the uncertainty of the free surface based on the method of Phillips and Roy (2017) is presented with $p=2$ in the denominator of Eq. (18). This is done following the recommendation of the above authors. The uncertainty shown in second place, i.e. that due to Roy (2008) is computed with a variable factor of safety, and the error provided by the error constants, shown in Eq. (11) and Eq. (12) multiplied by the fine grid spacing and the FS shown in Figure 16. This is then divided by $r^{p_{f}}-1$ in line with other error estimators. 

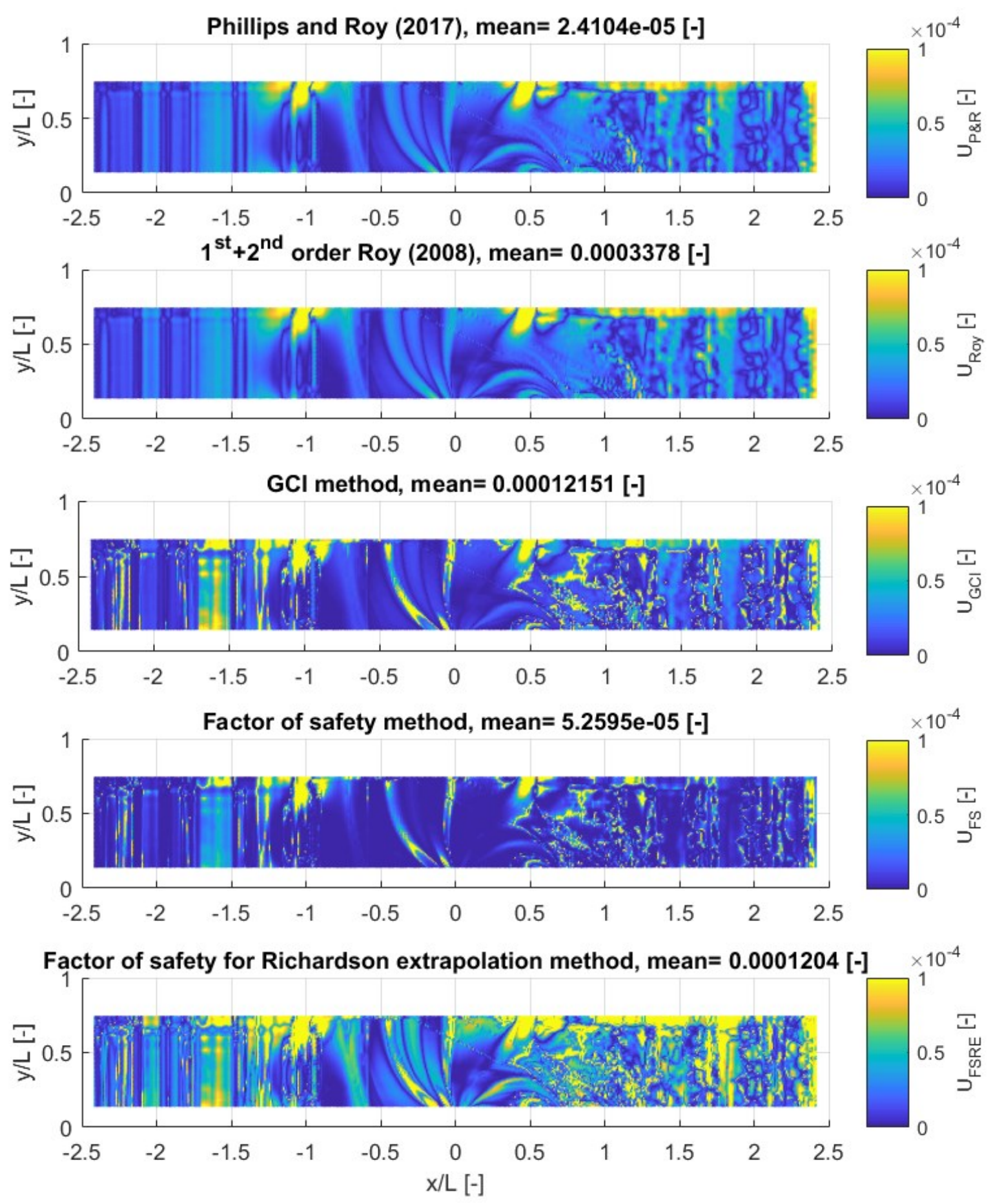

Figure 17. Local uncertainty spatial distribution on the free surface according to all methods.

The remainder of uncertainty estimators of Figure 17 follow as explained in Section 2. In other words, no modifications have been attempted on these. This is done to avoid compromising the confidence level of each prediction. The main conclusion, drawn from Figure 17 is that the first two methods, due to Phillips and Roy (2017) and due to Roy (2008) exhibit similar levels of convergence, in line with other methods with a longer history of implementation. This is a promising outcome, demonstrating that their use is likely capable of providing good results. Coupling this with the in-depth assessment possible based on the abovementioned methods makes them a desirable approach to error and uncertainty estimation. 
As postulated earlier, the locations of heightened uncertainty do not change across methods. However, the magnitude of the uncertainty is highly sensitive to the method employed. For instance, the GCI method predicts heightened uncertainty near $y / L$ between -2 and -1.5 . Although the decomposed linear and quadratic components also exhibit increased uncertainty in the vicinity of this region, error cancellation seems to dampen the uncertainty magnitude. Therefore, a greater level of detail can be extracted by the decomposed form of the error. Moreover, the uncertainty is highest in this type of error estimator. However, it should be employed with caution, because as stated previously, its confidence level has yet to be established. Based on the data generated as part of this study, the level of conservativeness predicted for the mixed order method is circa $51.64 \%$.

Conservativeness is defined as the percentage of points, where $f_{i}-U<f_{\text {ext }}<f_{i}+U$ is satisfied. In other words, points where the predicted extrapolated solution lies within the uncertainty band. Since the mixed order method of Roy (2008) is designed for specific orders of magnitude, it is not capable of providing a sufficiently large band of uncertainty to accommodate an extrapolated solution with an observed order of accuracy far from $1^{\text {st }}$ and $2^{\text {nd }}$ powers of the grid spacing. Alternatively, the extrapolation itself may not be a good estimate if the observed order of accuracy is far from the pre-defined powers of the grid spacing. On the other hand, the method of (Phillips and Roy, 2017) provided a coverage of $73.60 \%$.

A confidence interval below $95 \%$ is to be expected, since nearly half of all points were not classified as 'Richardson nodes', which is a precondition for the procedure itself. It is likely that the Factor of Safety's value (FS $=3$ ) is in part the reason why $73.60 \%$ coverage is achieved, rather than a confidence interval equal to the fraction of Richardson nodes. If the present exercise were to be repeated on a structured solver, one may expect that a greater confidence level would be achieved. Unstructured solvers tend to deteriorate the convergence properties of the solution (Thomas and Langley, 2008). Due to the small number of skin friction samples, confidence bands are not predicted for $\tau_{w}$.

The skin friction uncertainty is shown in Figure 18 according to the same methods as done for the free surface. In this case, the limits of the uncertainty have been increased for two methods: Roy's (2008) and the FSRE method. This is done to accommodate the larger predictions provided by these estimators. The degree to which the grid is asymptotic, demonstrated in the previous section (Figure 16), has rendered the uncertainty prediction due to Phillips and Roy (2017) the smallest. Such behaviour is justified, because the near-wall grid was shown to be highly asymptotic of all methods. The difference between the abovementioned technique and the GCI method is that the former uses the theoretical order of accuracy. The GCI method on the other hand employs the locally observed order, which is in large parts of the domain larger than two. This can be consulted in Figure 15.

One final aspect of Figure 18 worth considering relates to the distinction of wet and dry areas of the hull. In all methods, the above distinction is clearly visible, with submerged points tending towards greater uncertainty. To provide a more intuitive representation of the uncertainty, Figure 19 is constructed to show the fine solution along with the uncertainty computed by each method. In the plot it is more evident which locations along the $x$-axis are culpable for greater uncertainty levels. It is also manifest that points located near the origin of the abscissa contribute virtually nothing to the overall levels of uncertainty. As stated previously, these points represent the dry parts of the ship hull. 
The representation used in Figure 19 highlights the existence of several locations where certain uncertainty estimators predict a significantly greater level of uncertainty than others. For example, the GCI method shows that few points in the stern area of the ship are characterised by large errors. On the other hand, Roy's (2008) method predicts a greater number of points behaving in this manner. This is suspected to be due to the interaction of error constants, used by the latter method. Since the GCI method does not distinguish between different orders of convergence in this respect, the decomposition approach can unveil greater detail. At this stage, no comment in terms of robustness is possible, due to the previously examined reasons.
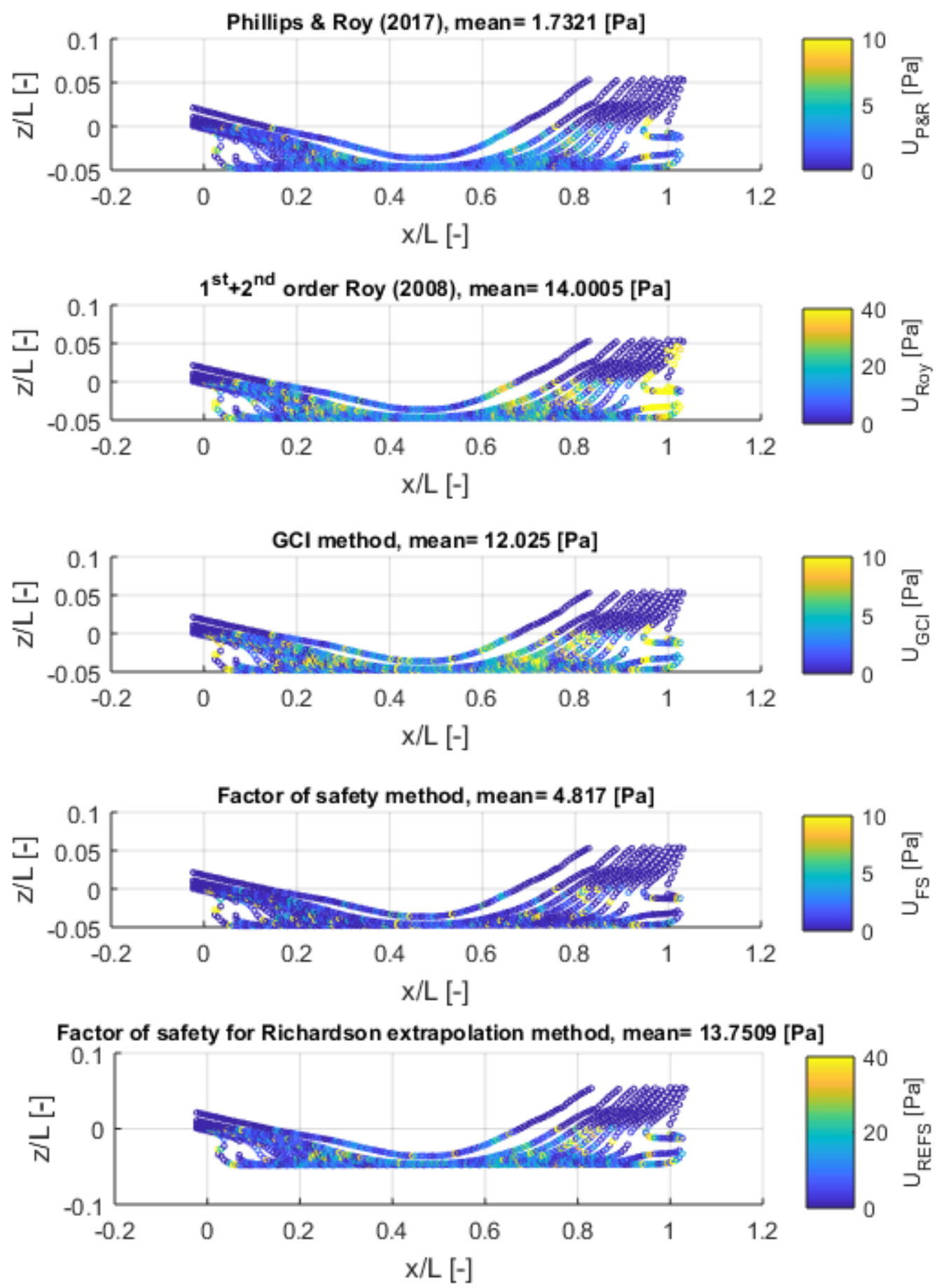
Figure 18. Local uncertainty spatial distribution skin friction on the ship hull according to all methods

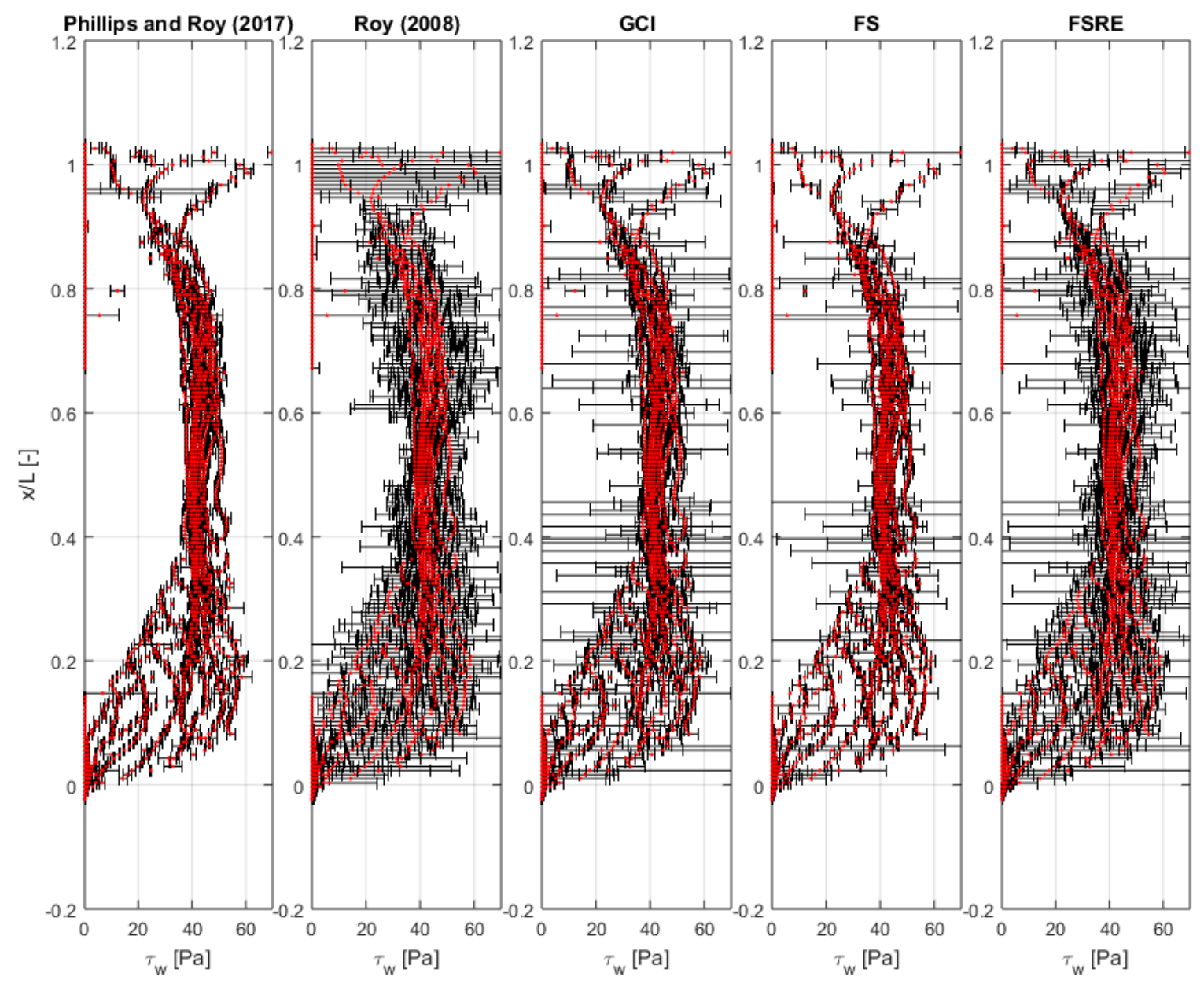

Figure 19. Skin friction with error bars.

\subsubsection{Extrapolated solutions}

This section examines the final aspect of the numerical verification procedure. Namely, the extrapolated solutions. There are two such solutions for each sampled point in each parameter (free surface and skin friction). These are the GCI and Roy's (2008) extrapolated solutions. A comparison between the two enables the analyst to confirm the locations of heightened error. Such locations will register in the extrapolated solution as large deviations from the fine solution. Furthermore, upon comparing extrapolated solutions achieved by different methods, it is possible to show which method can cope with non-monotonic input better. This is the case, because such a method would allow for oscillatory samples, providing an extrapolated solution that resembles the input to a greater extent.

For consistency, the free surface is examined first in Figure 20, where the two extrapolated solutions are accompanied by the ratio of the fine and respective extrapolated solution. This is done to better highlight the differences between the separate solutions, and their interaction with the fine solution. The figure serves to demonstrate that both methods predict a discontinuous line emanating from the aft perpendicular and progressing towards the centre of 
the domain. However, they disagree on its shape and extent. The same is true for a line in the forward part of the hull.
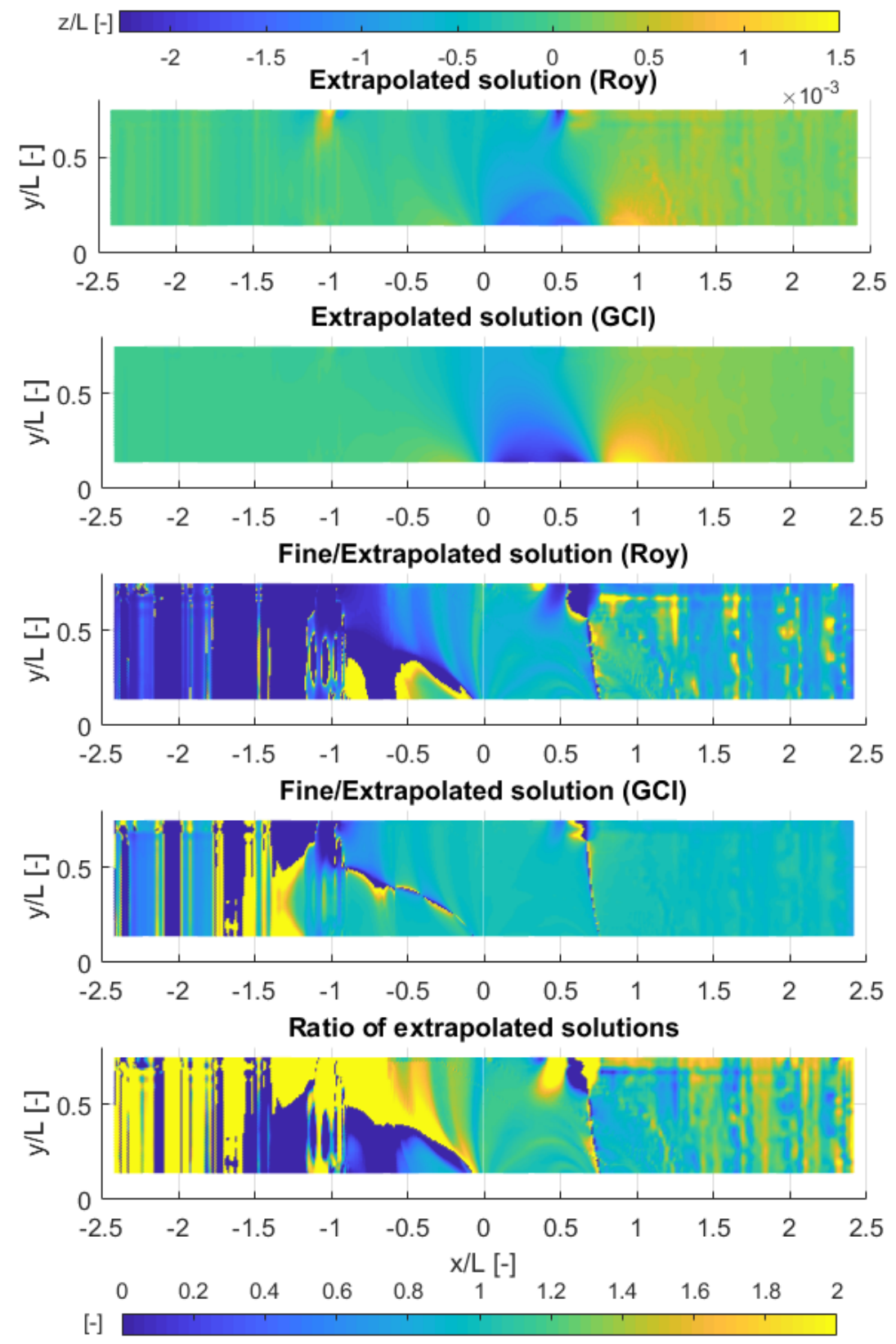

Figure 20. Extrapolated free surfaces

Figure 20 also reveals patches of differences forward of the ship, the origin of which can only be speculated at. The relative discrepancies between the two methods also highlight that the GCI method predicts to a lesser extent the semi-circular arcs in the proximity of the ship. The elevated levels of error at the side wall are also shown to be of different magnitude. More importantly, the region prescribed to provide the VOF damping seems to be a major source of disagreement between the two methods. This observation indicates that a wide range of numerical parameters simultaneously influence, and therefore compete for the dominant contribution in the error and uncertainty estimate. Splitting these from the grid-induced errors and bifurcating them as separate components may therefore not be the best approach in nonasymptotic grids. 
The next step is to examine how the two extrapolated solutions compare in terms of skin friction. This is depicted in Figure 21 in the same order as was previously done for the free surface. Here, the GCI method is shown to provide solutions that are practically indistinguishable from the fine solution itself. This can be verified by consulting the plot representing the ratio of the GCI solution and fine solution. Almost all points in this category have attained a value of either one, or are sufficiently close to one. The mixed order method on the other hand does not resemble the fine solution as closely. This causes differences in the predicted solution by the aforementioned approach to be evident when considering its ratio with the fine solution.

Upon comparing the ratio of the two extrapolated solutions in terms of skin friction, further differences emerge. These are visible primarily near the extremities of the ship, suggesting that areas of high curvature may cause disagreement between the two methods. It is likely that the cause of this observation once again stems from the interaction of linear and quadratic components. Figure 11 points towards the existence of large errors in both the linear and quadratic terms of the error in this region. Even a small error misalignment will create heightened levels of uncertainty, which may be responsible for the observed levels of disagreement in Figure 21.

In summary, this section demonstrated that each error and uncertainty estimator has a distinct tendency when it comes to predictions. The formulation of each distinct approach governs which regions of the sampled domain are highlighted with an increased concentration of numerical problems. In many cases, different aspects of the numerical solution may, and do interact in unpredictable ways. This may be the error itself, exhibiting discontinuities in terms of value as well as order, or other aspects, such as free surface modelling, convection properties or separate unexamined effects. These include sharpening factors in the VOF scheme, convection order, as well as the grid resolution. On the other hand, the interplay of cell aspect ratio, skewness angle, $y^{+}$value, turbulence model, and convection scheme order generate a distinct problem. In many cases, this interplay may be fortuitously favourable, but unknown, as is probably the case in terms of skin friction. On the other hand, the level of complexity of all components influencing the free surface make it impossible to accurately pinpoint the specific cause-effect relationship, leading to the observed error and uncertainty. However, by attempting to isolate separate aspects of the numerical solution, such as grid density, it is possible to provide recommendations relating to best practices. 

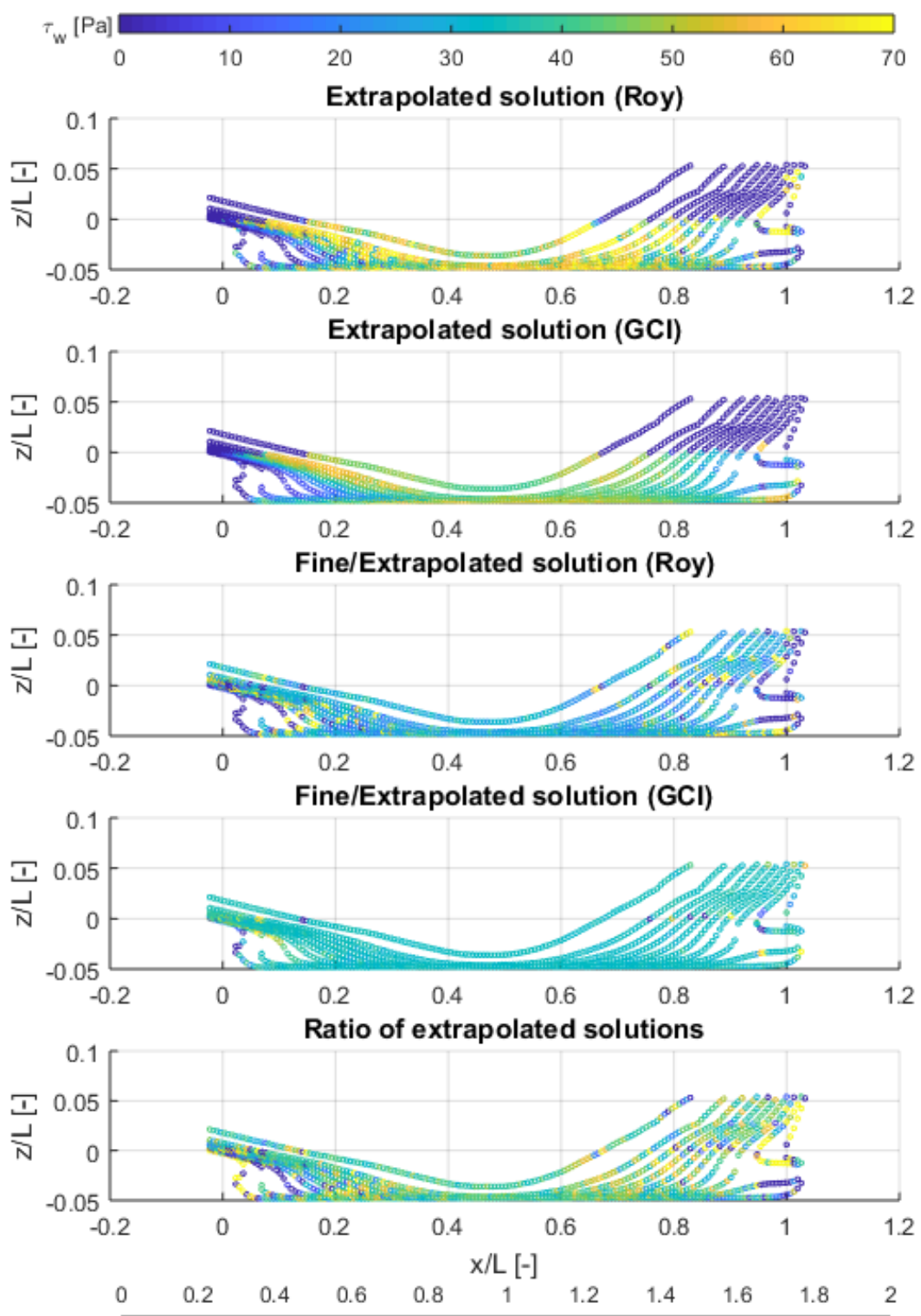

$[-]$

Figure 21. Extrapolated skin friction distribution on the hull.

The present study has demonstrated that in full-scale applications, a $y^{+}$value below one is not a necessary precondition to classifying the near-wall grid as asymptotic. On the other hand, a highly dense grid, encompassing the area where the free surface deforms was shown to be insufficient to brand the sampled points as generated by an asymptotic grid. This also highlights the fact that depending on the grid arrangement and numerical set-up, vastly different behaviours in terms of error can be achieved in separate aspects of the simulation. It should be borne in mind that an asymptotic solution does not necessarily translate into a low validation error. Therefore, the next step would be to compare the solution, generated by a study similar to that presented herein, with experimental data. Recently emerging methods to capture free surface deformations experimentally show potential in this respect (Caplier et al., 2016).

\subsection{Effect on sampling density}

The present work used $25 \times 10^{4}$ points to sample the free surface. It is not reasonable to expect such level of detail be used in industrial applications. This is the case for two reasons. Primarily, 
because exporting $25 \times 10^{4}$ points requires both time and care. But also, one would ideally like to know what is the minimal sample density required to produce a reliable result. This Section will attempt to answer the above question by taking a different number of samples, randomly from the available points.

The present sampling study is performed in steps. Firstly, statistical measures are used to determine the error due to sample size, assuming the exact number of Richardson nodes is known. Then, the available free surface is randomly sampled repeatedly at different levels of density.

The error due to sample size $(E)$ is estimated as shown in Eq. (21) for a 95\% confidence level, which is depicted graphically in Figure 22.

$E=1.96 \times \sqrt{m \times(m-1) / n}$

where $m$ is the currently estimated percentage of Richardson nodes $(43.9696 \%$ of all free surface points), and $n$ is the total number of available points. This procedure implicitly assumes that the above fraction of the total represents the true number of Richardson nodes in the domain. Such an assertion should be made with caution, because there is no manner to determine the exact number of nodes in each category. Moreover, the classification of nodes may change if the grid is refined further

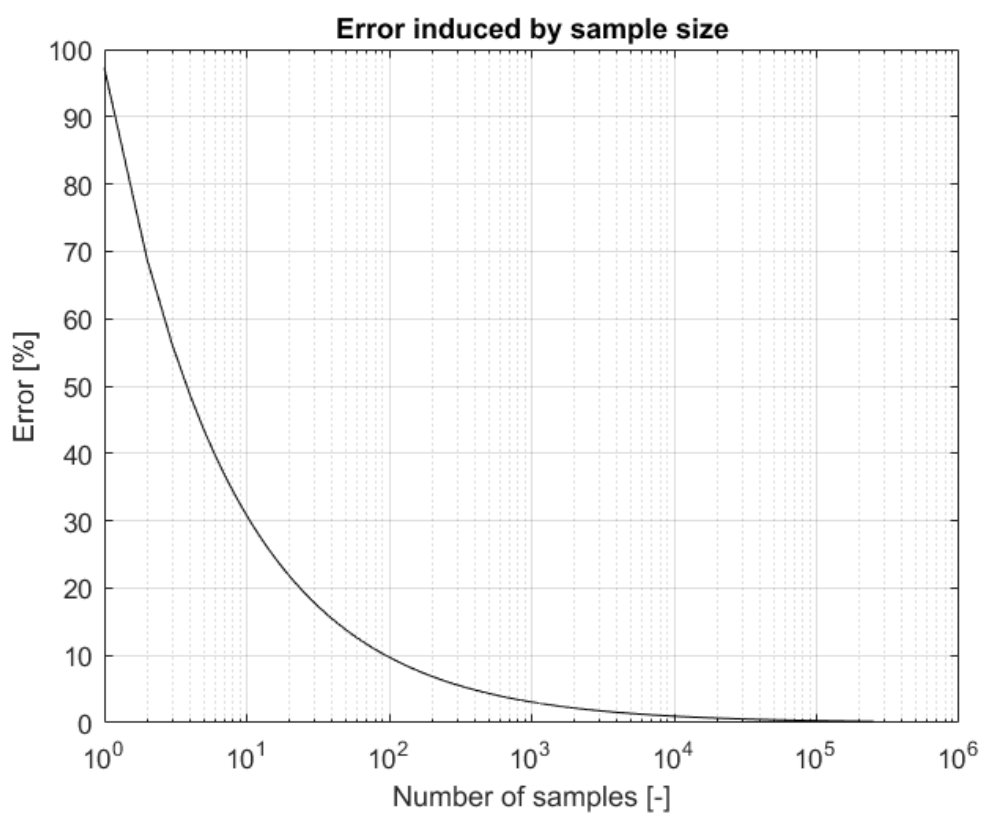

Figure 22. Error induced by sample size as predicted by Eq. (21).

For the reasons explained above, the free surface is also sampled using random, uniformly distributed points $25 \times 10^{4}$ times. This is performed at the density depicted in the title of each sub-plot of Figure 23, i.e. by taking $[50 \%, 25 \%, 12.5 \%, 6.25 \%, 3 \%, 1 \%, 0.1 \%, 0.01 \%]$ of all samples. The results from this study are presented in Figure 24. Here, the Factor of safety and fraction of Richardson nodes are monitored at each sampling iteration. Then, the standard deviation and mean values are used to construct the plot. No individual sample revealed a FS value other than 3, which is why no standard deviation of the Factor of Safety is included. The only varying metric was determined as the \% of Richardson nodes. The sample independent solution is also estimated in Figure 24 using the GCI procedure to extrapolate a point, based 
on the final three solutions. Since these exhibit oscillatory convergence, the absolute value modification is employed in the estimation of the order of convergence. The sampleindependent solution is shown as a filled circle at the end of the abscissa.

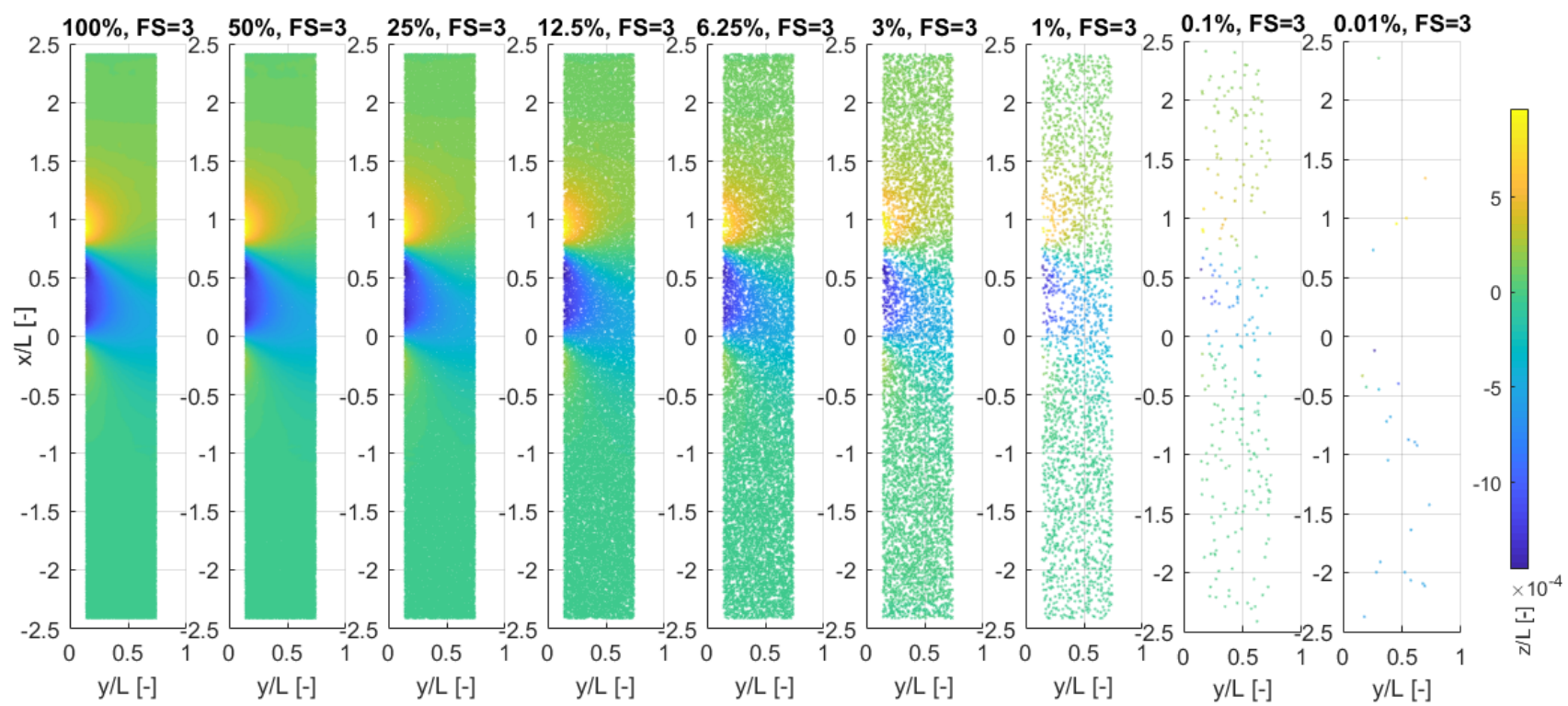

Figure 23. Sampling of the free surface

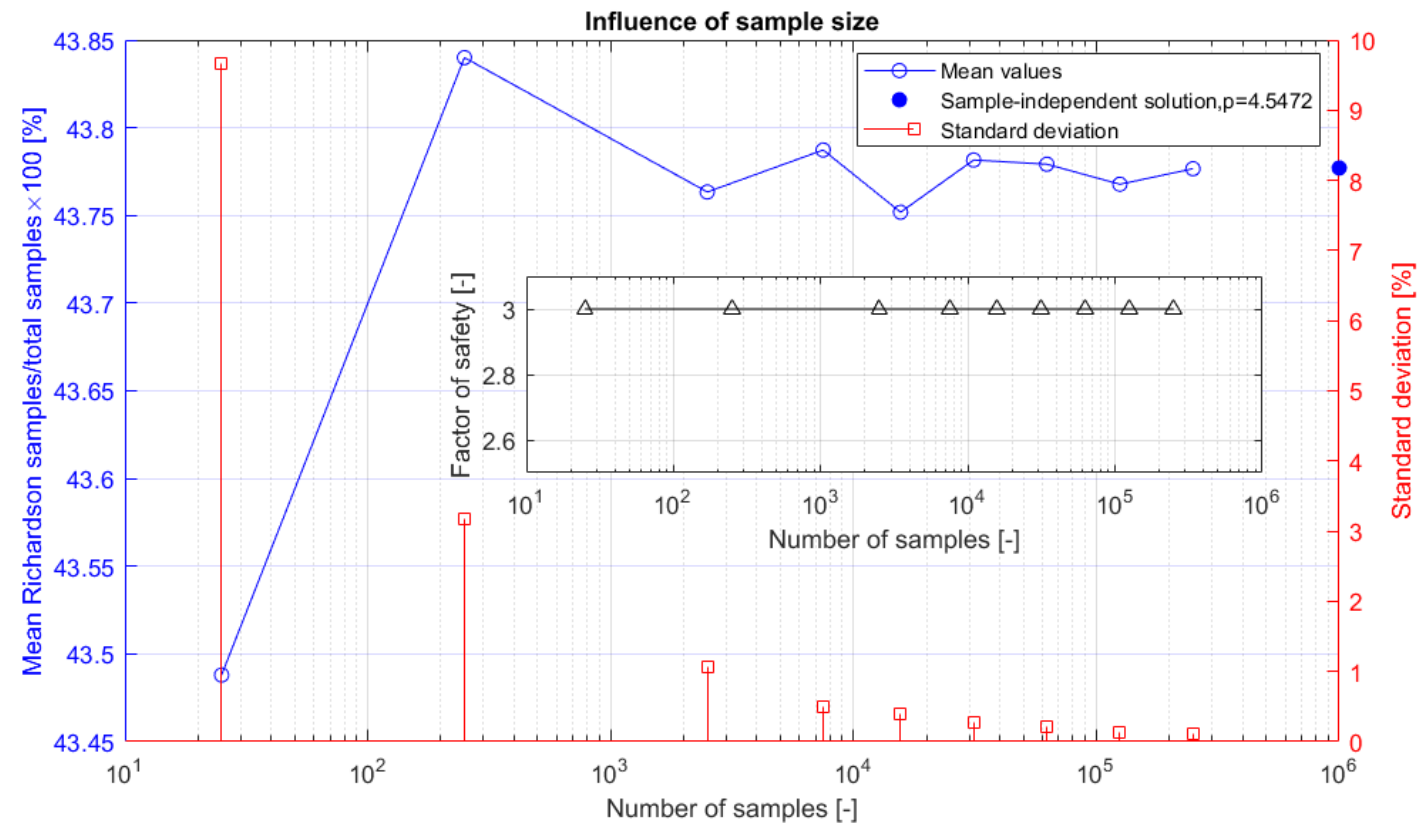

Figure 24. Influence of sample size.

The results presented in this Section suggest that $10^{4}$ samples should be sufficient to estimate the Factor of Safety and fraction of Richardson nodes with sufficient confidence. The first part of the assessment performed here suggests that such a sampling density would induce errors smaller than $1 \%$. The second assessment agrees with this conclusion, showing a standard deviation below $1 \%$ in the region of $10^{4}$ samples. Since both types of assessments show similar behaviour, it is concluded that the aforementioned number of samples in full-scale is sufficient to accurately estimate the parameters of interest.

\section{Conclusion and recommendations for future work}


The importance of verification of CFD works has gained increased importance and attention, with many influential academic journals and institutes revising their editorial policies to reject any papers not featuring such assessments (American Institute of Aeronautics and Astronautics (AIAA), 1994; Celik et al., 2008; Roache et al., 1986). This fact, coupled with the increased reliance on simulation-based design in all fields of engineering suggests that numerical verification will only increase in importance. It is therefore paramount that new methods are assimilated within all fields of engineering soon after their inception.

This study presented the application of non-intrusive a posteriori methods to assess the numerical performance of a set of CFD simulations. The main goal was to introduce some wellknown local methods of numerical verification to the field of ship hydrodynamics. To increase the practical applicability of the work presented herein, a set of full-scale simulations of the KCS advancing in a canal were adopted. Then, the numerical verification procedures were applied on local parameters, specifically, the free surface elevation and the non-dimensional shear stress, i.e. the skin friction.

The obtained results were presented in terms of several parameters of interest, including the decomposed error, uncertainty as well as the extrapolated solutions. In the case of the free surface, the assessment revealed semi-circular arcs of heightened numerical uncertainty emanating from the hull. The numerical damping beach implemented aft of the ship was also identified as a definite source of uncertainty. More importantly, it was demonstrated that the numerical uncertainty predictions in the damping region were in disagreement between the examined methods. Thus, suggesting that numerous aspects of the numerical simulation likely compete for the dominant error contribution. The grid, containing the free surface was also demonstrated to be far from asymptotic. This is likely due to interaction of different numerical parameters involving the definition of the free surface, which may affect the observed order of convergence.

In terms of skin friction, the assessment revealed that the near-wall grid is highly asymptotic. Regardless of the proximity of the generated grid to the asymptotic range, several uncertainty estimators were tested. These revealed significant disagreements in the magnitude of the uncertainty depending on the adopted approach. Based on the above findings it can be stated that the uncertainty is expected to be highly dependent on the parameter used in its prediction. Different parts of the computational domain are governed by distinct numerical schemes and approaches, thereby increasing the complexity of the problem at hand significantly.

The viscous-dominated part of the domain, located near impermeable boundaries, can be refined sufficiently to bring it in line with the asymptotic range. That is even tough this particular part of the computational domain features uncertainties stemming from the choice of turbulence model, $y^{+}$strategy, as well as a phase interphase (to name but a few aspects of the solution). On the other hand, the free surface grid resolution, containing for the majority of the cells in the domain was not near the asymptotic range. The distinction of the grid's performance with respect to the asymptotic range is important, because verification procedures implicitly contain the above requirement. Therefore, the determined uncertainty is a function of grid performance in this respect.

In full-scale hydrodynamic simulations of ship flows, the effects of surface roughness are not negligible. Therefore, the present assessment was conducted under highly idealised conditions, and should be extended to incorporate fouling of different extents (Demirel et al., 2014). It 
would be beneficial to demonstrate whether the asymptotic behaviour of the near-wall grid is maintained under different conditions applied to the surface of the ship. Since this would entail additional numerical procedures (Demirel et al., 2017), it is expected that accounting for roughness will have a detrimental effect on the convergence properties of the grid in this region, as well as globally. However, this needs to be demonstrated separately.

Another more complex problem would be the inclusion of self-propulsion. Allowing for propeller effects generates an unsteady field, which would also impact the free surface. Therefore, the samples must be generated with greater care and consideration. Skin friction on the propeller blades themselves are a further area of study the present assessment could be applied to. The influence of input parameter must also be established. This would determine which metric (velocity, pressure, etc.) is the most representative of the domain characteristics. There is also no reason why this study cannot be extended to encompass temporal discretisation effects. In other words, the time-step could be varied for a constant grid, with sampling performed at each distinct time-step. 


\section{Acknowledgements}

Results were obtained using the ARCHIE-WeSt High Performance Computer (www.archiewest.ac.uk) based at the University of Strathclyde. The work reported in this paper is drawn from the first author's $\mathrm{PhD}$ thesis. The first author gratefully acknowledges the scholarship provided by the Faculty of Engineering at the University of Strathclyde, which fully supports his $\mathrm{PhD}$.

\section{References}

American Institute of Aeronautics and Astronautics (AIAA), 1994. Editorial policy statement on numerical accuracy and experimental uncertainty. AIAA J. 32, 3. https://doi.org/10.2514/3.48281

Andrun, M., Blagojević, B., Bašić, J., 2018. The influence of numerical parameters in the finite-volume method on the Wigley hull resistance. Proc. Inst. Mech. Eng. Part M J. Eng. Marit. Environ. https://doi.org/10.1177/1475090218812956

Cadafalch, J., Pérez-Segarra, C.D., Cònsul, R., Oliva, A., 2002. Verification of finite volume computations on steady-state fluid flow and heat transfer. J. Fluids Eng. Trans. ASME 124, 11-21. https://doi.org/10.1115/1.1436092

Caplier, C., Rousseaux, G., Calluaud, D., David, L., 2016. Energy distribution in shallow water ship wakes from a spectral analysis of the wave field. Phys. Fluids 28. https://doi.org/10.1063/1.4964923

Celik, I.B., Ghia, U., Roache, P.J., Freitas, C.., 2008. Procedure for Estimation and Reporting of Uncertainty Due to Discretization in CFD Applications. J. Fluids Eng. 130, 078001. https://doi.org/10.1115/1.2960953

Celik, I.B., Karatekin, O., 1997. Numerical Experiments on Application of Richardson Extrapolation With Nonuniform Grids. J. Fluids Eng. 119, 584-590. https://doi.org/10.1115/1.2819284

Celik, I.B., Li, J., Hu, G., Shaffer, C., 2005. Limitations of Richardson Extrapolation and Some Possible Remedies. J. Fluids Eng. 127, 795. https://doi.org/10.1115/1.1949646

Demirel, Y.K., Khorasanchi, M., Turan, O., Incecik, A., Schultz, M.P., 2014. A CFD model for the frictional resistance prediction of antifouling coatings. Ocean Eng. 89, 21-31. https://doi.org/10.1016/j.oceaneng.2014.07.017

Demirel, Y.K., Turan, O., Incecik, A., 2017. Predicting the effect of biofouling on ship resistance using CFD. Appl. Ocean Res. 62, 100-118. https://doi.org/10.1016/j.apor.2016.12.003

Deng, G., Duvigneau, R., Queutey, P., Visonneau, M., 2004. Assessment of turbulence models for ship flow at full scale, in: Comp. Mech., WCCM VI. Beijing, China.

Diskin, B., Thomas, J.L., 2010. Notes on accuracy of finite-volume discretization schemes on irregular grids. Appl. Numer. Math. 60, 224-226. https://doi.org/10.1016/j.apnum.2009.12.001

Durbin, P.A., Pettersson Reif, B.A., 2011. Statistical theory and modelling for turbulent flow, Second Edi. ed. Wiley.

Eca, L., Hoekstra, M., 2009. Evaluation of numerical error estimation based on grid refinement 
studies with the method of the manufactured solutions. Comput. Fluids 38, 1580-1591. https://doi.org/10.1016/j.compfluid.2009.01.003

Eca, L., Hoekstra, M., 2006. Discretization Uncertainty Estimation based on a Least Squares version of the Grid Convergence Index. Proc. Second Work. CFD Uncertain. Anal. 1-27.

Eca, L., Hoekstra, M., 2001. Numerical Prediction of Scale Effects in Ship Stern Flows with Eddy-Viscosity Turbulence Models, in: Twenty-Second Symposium on Naval Hydrodynamics Office of Naval Research Bassin d'Essais Des CarenesNational Research Council. pp. 553-568. https://doi.org/10.17226/9771

Eca, L., Hoekstra, M., Beja Pedro, J.F., Falcao de Campos, J.A.C., 2013. On the characterization of grid density in grid refinement studies for discretization error estimation. Int. J. Numer. Methods Fluids 72, 119-134. https://doi.org/https://doi.org/10.1002/fld.3737

Eca, L., Saraiva, G., Vaz, G., Abreu, H., 2015. The Pros and Cons of Wall Functions. OMAE2015, St.Johns, Newfoundland, Canada 0-11. https://doi.org/10.1115/OMAE2015-41518

Elsherbiny, K., Tezdogan, T., Kotb, M., Incecik, A., Day, S., 2019. Experimental analysis of the squat of ships advancing through the New Suez Canal. Ocean Eng. 178, 331-344. https://doi.org/10.1016/j.oceaneng.2019.02.078

Freitas, C.J., 2002. The issue of numerical uncertainty. Appl. Math. Model. 26, 237-248. https://doi.org/10.1016/S0307-904X(01)00058-0

Fureby, C., Toxopeus, S.L., Johansson, M., Tormalm, M., Petterson, K., 2016. A computational study of the flow around the KVLCC2 model hull at straight ahead conditions and at drift. Ocean Eng. 118, 1-16. https://doi.org/10.1016/j.oceaneng.2016.03.029

Hawkes, J., Vaz, G., Phillips, A.B., Cox, S.J., Turnock, S.R., 2018. On the strong scalability of maritime CFD. J. Mar. Sci. Technol. 23, 81-93. https://doi.org/10.1007/s00773-0170457-7

ITTC, 2014. ITTC - Recommended Procedures and Guidelines - Practical guidelines for ship CFD applications. 7.5-03-02-03 (Revision 01). ITTC - Recomm. Proced. Guidel. 19.

ITTC, 2011. Recommended Procedures and Guidelines: Practical Guidelines for Ship CFD. 26th Int. Towing Tank Conf.

ITTC, 2002. ITTC - Recommended Procedures and Guidelines Uncertainty Analysis , Example for Resistance Test.

Jasak, H., 1996. Error analysis and estimation for finite volume method with applications to fluid flow. PhD Thesis. Imperial College of Science, Technology and Medicine.

Kornev, N., Abbas, N., 2018. Vorticity structures and turbulence in the wake of full block ships. J. Mar. Sci. Technol. 23, 567-579. https://doi.org/10.1007/s00773-017-0493-3

Kornev, N., Shevchuk, I., Abbas, N., Anschau, P., Samarbakhsh, S., 2019. Potential and limitations of scale resolved simulations for ship hydrodynamics applications. Sh. Technol. Res. 7255. https://doi.org/10.1080/09377255.2019.1574965

Larsson, L., Stern, F., Visonneau, M., 2014. Numerical Ship Hydrodynamics: An assessment 
of the Gothenburg 2010 Workshop. Springer. https://doi.org/10.1007/978-94-007-7189-5

Lax, P.D., Richtmyer, R.D., 1956. Survey of the stability of linear finite difference equations. Commun. Pure Appl. Math. 9, 267-293. https://doi.org/10.1002/cpa.3160090206

Liefvendahl, M., Fureby, C., 2017. Grid requirements for LES of ship hydrodynamics in model and full scale. Ocean Eng. 143, 259-268. https://doi.org/10.1016/j.oceaneng.2017.07.055

Lilek, Ž., Peric, M., 1995. A fourth-order finite volume method with colocated variable arrangement. Comput. Fluids 24, 239-252. https://doi.org/10.1016/0045-7930(94)000303

Morton, K.W., Mayers, D.F., 2005. Numerical Solution of Partial Differential Equations: An Introduction. Cambridge University Press, Cambridge. https://doi.org/10.1017/CBO9780511812248

Oberkampf, W.L., Blottner, F.G., 1998. Issues in computational fluid dynamics code verification and validation. AIAA J. 36, 687-695. https://doi.org/10.2514/2.456

Parneix, S., Durbin, P.A., Behnia, M., 1998. Computation of 3-D turbulent boundary layers using the V2F model. Flow, Turbul. Combust. 60, 19-46. https://doi.org/10.1023/A:1009986925097

Perić, R., Abdel-Maksoud, M., 2016. Reliable damping of free-surface waves in numerical simulations. Sh. Technol. Res. 63, 1-13. https://doi.org/10.1080/09377255.2015.1119921

Phillips, T., 2014. Residual-based Discretization Error Estimation for Computational Fluid Dynamics 148.

Phillips, T., 2012. Extrapolation-based Discretization Error and Uncertainty Estimation in Computational Fluid Dynamics.

Phillips, T., Roy, C.J., 2017. A New Extrapolation-Based Uncertainty Estimator for Computational Fluid Dynamics. J. Verif. Valid. Uncertain. Quantif. 1, 041006. https://doi.org/10.1115/1.4035666

Phillips, T., Roy, C.J., 2012. Evaluation of Extrapolation-Based Discretization Error and Uncertainty Estimators, AIAA 2011-215. 49th AIAA Aerosp. Sci. Meet. Incl. New Horizons Forum Aerosp. Expo. Orlando, F, 1-18. https://doi.org/10.2514/6.2011-215

Richardson, L.F., 1927. Deferred approach to the limit. Trans. R. Soc. London, Ser. A 226, 299-361.

Richardson, L.F., 1911. The Approximate Arithmetical Solution by Finite Differences of Physical Problems involving Differential Equations, with an Application to the Stresses in a Masonry Dam. Philos. Trans. th R. Soc. London,Containing Pap. a Math. Phys. Character 210, 307-357.

Roache, P.J., 1998. Validation and Verification in computational science and engineering. Hermosa Albuquerque, NM.

Roache, P.J., 1997. Quantification of Uncertainty in Computational Fluid Dynamics. Annu. Rev. Fluid Mech. 29, 123-160. https://doi.org/10.1146/annurev.fluid.29.1.123

Roache, P.J., Ghia, K.N., White, F., 1986. Editorial Policy Statement on the Control of Numerical Accuracy. J. Fluids Eng. 108, 2. 
Roache, P.J., Knupp, P.M., 1993. Completed richardson extrapolation. Commun. Numer. Methods Eng. 9.

Roy, C.J., 2008. Grid Convergence Error Analysis for Mixed-Order Numerical Schemes. AIAA J. 41, 595-604. https://doi.org/10.2514/2.2013

Roy, C.J., 2005. Review of code and solution verification procedures for computational simulation. J. Comput. Phys. 205, 131-156. https://doi.org/10.1016/j.jcp.2004.10.036

Roy, C.J., Blottner, F.G., 2006. Review and Assessment of Turbulence Models for Hypersonic Flows: 2D/Asymmetric Cases. 44th AIAA Aerosp. Sci. Meet. Exhib. https://doi.org/10.2514/6.2006-713

Salas, M.D., 2006. Some observations on grid convergence. Comput. Fluids 35, 688-692. https://doi.org/10.1016/j.compfluid.2006.01.003

Salas, M.D., Atkins, H.L., 2009. Problems associated with grid convergence of functionals. Comput. Fluid Dyn. 2008 38, 309-314. https://doi.org/10.1016/j.compfluid.2008.01.015

Shevchuk, I., Kornev, N., 2017. Study of unsteady hydrodynamic effects in the stern area of river cruisers in shallow water. 7th Int. Conf. Comput. Methods Mar. Eng. Mar. 2017 2017-May, 440-448. https://doi.org/10.1080/09377255.2017.1349599

Siemens, 2018. Star-CCM+ User Guide version 13.04.

Slotnick, J., Khodadoust, A., Alonso, J., Darmofal, D., Gropp, W., Lurie, E., Mavriplis, D., 2014. CFD Vision 2030 Study: A Path to Revolutionary Computational Aerosciences, NASA/CR-2014-218178. https://doi.org/10.1017/CBO9781107415324.004

Stern, F., Wilson, R. V., Coleman, H.W., Paterson, E.G., 2001. Comprehensive approach to verification and validation of CFD simulations-Part 1: Methodology and procedures. J. Fluids Eng. Trans. ASME 123, 793-802. https://doi.org/10.1115/1.1412235

Terziev, M., Tezdogan, T., Incecik, A., 2019. Application of eddy-viscosity turbulence models to problems in ship hydrodynamics. Ships Offshore Struct. 1-24. https://doi.org/10.1080/17445302.2019.1661625

Terziev, M., Tezdogan, T., Oguz, E., Gourlay, T., Demirel, Y.K., Incecik, A., 2018. Numerical investigation of the behaviour and performance of ships advancing through restricted shallow waters. J. Fluids Struct. 76, 185-215. https://doi.org/10.1016/j.jfluidstructs.2017.10.003

Tezdogan, T., Demirel, Y.K., Kellett, P., Khorasanchi, M., Incecik, A., Turan, O., 2015. Fullscale unsteady RANS CFD simulations of ship behaviour and performance in head seas due to slow steaming. Ocean Eng. 97, 186-206. https://doi.org/10.1016/j.oceaneng.2015.01.011

Tezdogan, T., Incecik, A., Turan, O., 2016. A numerical investigation of the squat and resistance of ships advancing through a canal using CFD. J. Mar. Sci. Technol. 21, 86101. https://doi.org/10.1007/s00773-015-0334-1

Thomas, J.L., Langley, N., 2008. Toward Verification of Unstructured-Grid Solvers. AIAA J. 46. https://doi.org/10.2514/1.36655

Vanka, S.P., 1987. Second-order upwind differencing in a recirculating flow. AIAA J. 25, 1435-1441. https://doi.org/10.2514/3.9801 
Visonneau, M., 2005. A Step Towards the Numerical Simulation of Viscous Flows Around Ships at Full-Scale. Mar. CFD.

Witherden, F.D., Jameson, A., 2017. Future directions of computational fluid dynamics, in: 23rd AIAA Computational Fluid Dynamics Conference, 2017. pp. 1-16.

Xing, T., Stern, F., 2010. Factors of Safety for Richardson Extrapolation. J. Fluids Eng. 132, 061403. https://doi.org/10.1115/1.4001771

Xu, X., Li, H.A.O., Lin, Y., 2019. Mesh - Order Independence in CFD Simulation. IEEE Access 7, 119069-119081. https://doi.org/10.1109/ACCESS.2019.2937450

Zhang, S., Tezdogan, T., Zhang, B., Xu, L., Lai, Y., 2018. Hull form optimisation in waves based on CFD technique. Ships Offshore Struct. 13, 149-164. https://doi.org/10.1080/17445302.2017.1347231 